\title{
Discrete Output Regulator Design for the Linearized Saint-Venant-Exner Model
}

\author{
Guilherme Ozorio Cassol (1) and Stevan Dubljevic* *(1) \\ Department of Chemical \& Materials Engineering, University of Alberta, Edmonton, AB T6G 2V4, Canada; \\ ozorioca@ualberta.ca \\ * Correspondence: Stevan.Dubljevic@ualberta.ca; Tel.: +1-780-248-1596; Fax: +1-780-492-2881
}

Received: 25 June 2020; Accepted: 24 July 2020; Published: 1 August 2020

\begin{abstract}
This manuscript addresses the regulator design in the discrete-time setting for the unstable linearized Saint-Venant-Exner model, which describes the dynamics of a sediment-filled water canal. The proposed regulator ensures the closed-loop stability and proper tracking of polynomial and periodic reference signals using output feedback in a sample-data setting. To design this regulator, the system discrete representation is achieved by the application of the structure-preserving Cayley-Tustin time discretization and the direct relation with the regulator in the continuous-time setting is shown. The regulator design in the continuous-time setting is developed using the backstepping methodology ensuring the closed-loop stability and the observer design, while the Sylvester equations are solved to achieve proper tracking. Finally, the numerical simulation results are presented to show the performance of the regulator.
\end{abstract}

Keywords: system of hyperbolic PDEs; discrete regulator; output regulation; tracking

\section{Introduction}

In many chemical, hydro-geological, petrochemical, and manufacturing processes the dynamic models take the mathematical form of partial differential equations (PDEs). One of these types of models is the Saint-Venant-Exner (SVE) equations, which consist of non-linear PDEs and are used to model the dynamics of a sediment-filled water canal with arbitrary values of the bottom slope, friction, porosity, and water-sediment interaction. Due to their nature, one of the crucial aspects of these distributed parameter systems is the complexity given by the infinite-dimensional system representation [1,2], which is a challenging factor when it comes to the controller/regulator design and realization.

The primary goal of a regulator is to drive the desired output of a system to behave exactly as demanded, and at the same time it needs to assure the stability of the closed-loop system. Using early lumping methods is the simplest way to design the controller for distributed parameter systems. In these methods, the PDEs are generally converted to sets of ordinary differential equations (ODEs), which allows the use of standard control methodology applicable to ODE systems. But, this also results in some mismatch between the dynamical properties of the original distributed parameter and the lumped parameter models, thus, affecting the controller [3].

Another way to deal with distributed parameter processes is to exploit the infinite-dimensional characteristic of the system, and there are significant research efforts made to solve the regulator design problem for infinite-dimensional systems [4,5]. In [6], the generalized geometric methods were introduced in the regulator design for the first-order hyperbolic PDEs, and the robust output regulation problems were considered in [7-9], while taking into account infinite-dimensional exogenous systems (an independent system responsible for the generation of the tracking signal and/or disturbance). Specifically for the SVE model, there are several contributions that take into account the characteristics of the model. In [10], the proportional and integral output feedback controllers were proposed by 
using the semigroup theory. The $H_{\infty}$ optimization framework was applied in [11] to design a controller considers the both water resource management and performance with respect to the users. And in [12], a Lyapunov approach was used to obtain the control laws that stabilize the system considering full-state and output feedback.

More recently, in [13], the exponential stabilization of this model was achieved by the backstepping design, also considering full-state and output feedback, where the design of an exponentially stable Luenberger observer was considered for state reconstruction. The PDE backstepping design has proved to be of valuable for the boundary stabilization of distributed parameter systems. Essentially, the technique consists of finding a suitable transformation that maps the closed-loop system into a stable target system. Due to the invertibility of the transformation, the original and the target system have equivalent stability $[14,15]$. Other applications and developments along this line include, for instance, the boundary observer based-control design for a hyperbolic PDE [16], the boundary observer for a class of time-varying linear hyperbolic partial integral-differential equations (PIDEs) [17], and control of general linear heterodirectional hyperbolic ODE-PDE-ODE systems [18].

All the contributions mentioned above were developed in the continuous-time domain, but, as most modern and state-of-the-art controller realizations are digital and discrete, time discretization realizations need to be taken into account at the final design stage. Although some contributions addressed the stabilization of PDEs using backstepping with time sampled-data $[19,20]$, these were made considering specific scalar equations and the design of a controller in the discrete-time setting was not the objective of these works. Traditional time discretization schemes (for instance, explicit or implicit Euler) could be used to obtain a discrete-time representation, but they have the disadvantage of reducing the accuracy of the discrete system representation as the sampling period increases [21]. Moreover, the sampling may impact the overall model and closed-loop stability when the controller is implemented. Therefore, a different type of time discretization scheme, that provides a reliable transformation of a continuous linear infinite-dimensional system representation to a linear discrete-time infinite-dimensional needs to be considered. A discretization scheme that accounts for this design criteria is the Crank-Nicolson midpoint integration rule, which can be easily applied to infinite-dimensional systems [22]. This type of discretization is also known as Cayley-Tustin time discretization, and it has been shown to preserve the intrinsic energy and dynamical characteristics of the linear distributed parameter system [23] without the application of spatial discretization or/and model reduction.

The unstable linearized SVE model is considered in this manuscript. The PDE system is given as a system of first-order transport hyperbolic PDE equations, with in-domain and boundary coupling. Based on this system, a discrete-time output regulator design is presented and attains the following objectives: (1) ensures the stability by output feedback; (2) considers the stabilization of the problem in the discrete-time setting, obtained by the application of the Caley-Tustin time discretization; (3) achieves tracking of periodic and polynomial signals generated by an exosystem, which is ensured by the solution of the corresponding Sylvester output regulation equations. To properly design the discrete-time regulator, the relation between the discrete-time and continuous-time control is developed, such that the closed-loop stability and proper tracking of the discrete-time representation is assured if the controller design is known in the continuous-time.

The manuscript is organized as follows: in Section 2, the SVE system model and its properties are introduced together with the exosystem and control objectives. In Section 3, the system stabilization, observer design, and output regulation in the continuous-time setting are developed. In Section 4, the Caley-Tustin time discretization is applied to the system, and the discrete regulator design is developed. The stability of the closed-loop system is shown. In Section 5, the simulations results are presented and the regulator performance is discussed. Lastly, in Section 6, the final remarks are made.

\section{Problem Formulation}

The Saint-Venant and Exner equations are used to describe the dynamics in a sediment-filled open channel with rectangular cross-section [13]. Considering $H(t, \zeta)$ to be the water depth, $V(t, \zeta)$ 
as the water velocity and $B(t, \zeta)$ as the depth of the sediment layer above the channel bottom, the dynamics of the system can be described as the equations below:

$$
\begin{aligned}
& \frac{\partial H}{\partial t}+V \frac{\partial H}{\partial \zeta}+H \frac{\partial V}{\partial \zeta}=0 \\
& \frac{\partial V}{\partial t}+V \frac{\partial V}{\partial \zeta}+g \frac{\partial H}{\partial \zeta}+g \frac{\partial B}{\partial \zeta}=g S_{b}-C_{f} \frac{V^{2}}{H} \\
& \frac{\partial B}{\partial t}+a V^{2} \frac{\partial V}{\partial \zeta}=0
\end{aligned}
$$

As shown in [13], this system can be linearized around a steady-state and the Cardano-Vieta method can be applied to rewrite it in the characteristic form, which can be llustrated in Figure 1.

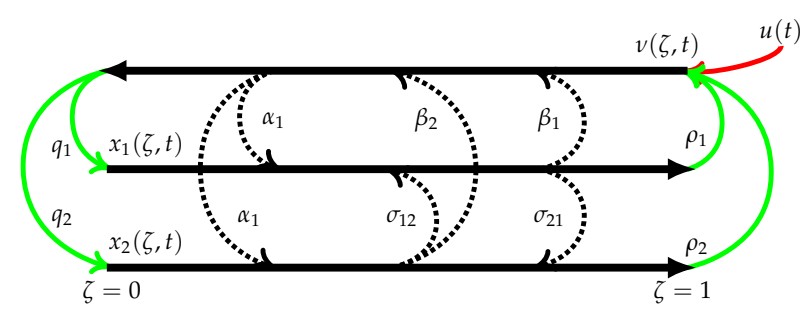

Figure 1. PDE system (Equation (2)) representation.

This linearized system is given by the following coupled system of first-order hyperbolic partial differential equations, in the domain $\left\{t \in \Re^{+}, \zeta \in[0,1]\right\}$, with $t$ representing time and $\zeta$ representing the dimensionless spatial variable:

$$
\begin{aligned}
& \frac{\partial v}{\partial t}=\mu \frac{\partial v}{\partial \zeta}+\alpha_{1} v+\beta_{1} x_{1}+\beta_{2} x_{2} \\
& \frac{\partial x_{1}}{\partial t}=-\gamma_{1} \frac{\partial x_{1}}{\partial \zeta}+\alpha_{1} v+\sigma_{11} x_{1}+\sigma_{12} x_{2} \\
& \frac{\partial x_{2}}{\partial t}=-\gamma_{2} \frac{\partial x_{2}}{\partial \zeta}+\alpha_{1} v+\sigma_{21} x_{1}+\sigma_{22} x_{2} \\
& y_{m}(t)=v(0, t) \\
& y(t)=x_{1}(1, t)
\end{aligned}
$$

With the following algebraic boundary conditions:

$$
\begin{aligned}
v(1, t) & =\rho_{1} x_{1}(1, t)+\rho_{2} x_{2}(\zeta=1, t)+u(t) \\
x_{1}(0, t) & =q_{1} v(0, t) \\
x_{2}(0, t) & =q_{2} v(0, t)
\end{aligned}
$$

These linear hyperbolic PDEs represent the transport of $x(\zeta, t)=\left[v, x_{1}, x_{2}\right]^{T} \in L^{2}(0,1)$ (a real Hilbert space); $\gamma_{1}, \gamma_{2}$ and $\mu$ are the system's characteristics velocities; $\sigma_{i j}$ for $i=1,2$ and $j=1,2, \alpha_{1}, \beta_{1}$ and $\beta_{2}$ are the parameters representing the in-domain interaction between the state variables; and $\rho_{1}$, $\rho_{2}, q_{1}$ and $q_{2}$ are parameters representing the interaction on the boundaries. All these parameters are obtained from the characteristic form of the SVE model [13].

Usually, the openings of the gates located at the ends of the channel can be controlled to achieve the stabilization of the water level and flow rate. Thus, $u(t) \in \Re$ (a real finite space), the system input, is considered to be control of the downstream gate, represented by the boundary actuation at $\zeta=1$, shown in (3). Measurements at the upstream $(\zeta=0)$ are considered to be the system measured output $y_{m}(t) \in \Re$ and are used to reconstruct the states with a Luenberger observer. The system desired 
system output $y(t) \in \Re$ is related to the properties of the water downstream. There are no direct measurements of this output and the regulator aim is to control it as desired. Thus, this system output should properly follow a predetermined pattern if the regulator is properly designed.

This system can be represented as an abstract differential equation:

$$
\begin{aligned}
& \dot{x}(t)=A x(t)+B u(t) \\
& y_{m}(t)=C_{m} x(t) \\
& y(t)=C x(t)
\end{aligned}
$$

where $A$ is a linear operator $\mathfrak{L}\left(L^{2}(0,1), L^{2}(0,1)\right), B$ is the linear input operator $\mathfrak{L}\left(\Re, L^{2}(0,1)\right), C_{m}$ is the measured output operator $\mathfrak{L}\left(L^{2}(0,1), \Re\right)$ and $C$ is the desired output operator $\mathfrak{L}\left(L^{2}(0,1), \Re\right)$.

In this contribution, the goal is to achieve proper tracking of a reference signal, while maintaining the system stability. It is considered that the reference signal $y_{r}(t) \in \Re$ to be tracked by the system output $y(t)$ is generated as the output of a known finite-dimensional exogenous system (also called exosystem), which is independent from the system (the exosystem affects the system dynamics, but not the other way around) and is defined as the following throughout this work:

$$
\begin{gathered}
\dot{z}(t)=S z(t), t>0, z(0) \in \mathbb{R}^{e} \\
y_{r}(t)=Q z(t), t \geq 0
\end{gathered}
$$

where the matrix $S: D(S): \mathbb{R}^{e} \rightarrow \mathbb{R}^{e}$ gives the dynamics of the exosystem states and $Q$ is a matrix that gives the desired output tracking signal $y_{r}(t)$.

Assumption 1. The reference signal consists of periodic and polynomial functions, such that the exosystem dynamics can be represented in the following form:

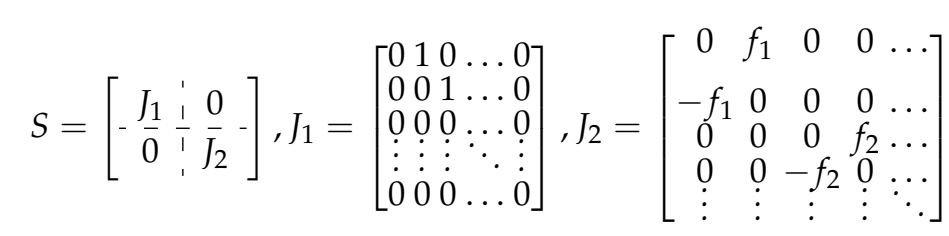

notice that $J_{1}$ and $J_{2}$ are decoupled block matrices and it is assumed that each $f_{i}$ is unique. $J_{1}$ generates a polynomial signal and the size of $J_{1}\left(n_{1}\right)$ determines the polynomial degree. $J_{2}$ generates a linear combination of periodic function, where each $f_{i}$ is responsible for a function with different periodicity. Therefore, the size of $J_{2}$ will be $n_{2}$, and it is an even number.

Remark 1. The block matrix $J_{1}$ has all eigenvalues equal zero (i.e., $\sigma\left(J_{1}\right)=0$ ) with multiplicity equal to the size of the matrix. $J_{2}$ on the other hand has conjugated complex eigenvalues $\sigma\left(J_{2}\right)=\left\{ \pm f_{i} \mathbf{j}\right\}$. Therefore, the spectra of $S$ in Equation (5) is $\sigma(S)=\sigma\left(J_{1}\right) \cup \sigma\left(J_{2}\right)=\left\{0, \pm f_{i} \mathbf{j}\right\}$. Due to their structures, $J_{1}$ is a Jordan block, while $J_{2}$ is a diagonal block matrix and its eigenvectors are filled with zeros in all rows except in the ones that correspond to each $f_{i}$. The elements in these rows will be $[1,-\mathbf{j}]^{T}$ and $[1,+\mathbf{j}]^{T}$ for the corresponding $\pm f_{i} \mathbf{j}$ eigenvalues.

This exosystem will generate a linear combination of a polynomial $\left(\sum_{i=0}^{n_{1}} a_{i} t^{i}\right.$ from $\left.J_{1}\right)$ and periodic functions with frequencies $f_{i}\left(\sum_{i=0}^{n_{2} / 2} b_{i} \sin \left(f_{i} t\right)+c_{i} \cos \left(f_{i} t\right)\right.$ from $\left.J_{2}\right)$, and $Q=\left[Q_{1}, Q_{2}\right]=$ $\left[Q_{1,1}, \ldots, Q_{1, n_{1}}, Q_{2,1}, \ldots, Q_{2, n_{2}}\right]$ and the initial conditions $z(0)$ are defined accordingly to match the desired tracking signal. Without loss of generality, one can extend the ensuing design to the case when the exosystem is infinite dimensional [9].

In this contribution, an exosystem that generates a first-order polynomial in combination a periodic signal with one frequency is considered. Therefore, the desired reference signal $\left(y_{r}(t)\right)$ has a general form given by: 


$$
y_{r}(t)=\underbrace{Q_{1,1} z_{1}(0)+\left(Q_{1,2}+Q_{1} t\right) z_{2}(0)}_{J_{1}}+\underbrace{\left[Q_{2,1} \cos (f t)-Q_{2,2} \sin (f t)\right] z_{3}(0)+\left[Q_{2,2} \cos (f t)+Q_{2,1} \sin (f t)\right] z_{4}(0)}_{J_{2}}
$$

with:

$$
J_{1}=\left[\begin{array}{ll}
0 & 1 \\
0 & 0
\end{array}\right], J_{2}=\left[\begin{array}{cc}
0 & f \\
-f & 0
\end{array}\right]
$$
signal:

The tracking error $e(t)$ is defined as the difference between the system output and the tracking

$$
e(t)=y(t)-y_{r}(t)
$$

In the continuous-time setting the regulation problem can be defined as finding a regulator of the form:

$$
u(t)=K[x(t)]+L z(t)
$$

where $K(\cdot)$ is the linear feedback gain operator $\mathfrak{L}\left(L^{2}(0,1), \Re\right)$ that is used to stabilize the system and $L$ is the feedforward gain $\mathfrak{L}\left(\mathbb{R}^{e}, \Re\right)$ that guarantees proper tracking of the desired signal. Therefore, the regulator should guarantee the following conditions:

- The closed-loop system is exponentially stable;

- For the closed-loop system, the tracking error $e(t \rightarrow \infty)=0, \forall x(0) \in X, z(0) \in \Re^{e}$;

\subsection{System Properties}

\subsubsection{Linearized System Stability}

The system stability can be determined by the analysis of the eigenvalue problem $A \psi(\zeta)=\lambda \psi(\zeta)$, where $\psi(\zeta)$ are the eigenvector of the system, in this case given by $\psi(\zeta)=\left[\psi_{1}(\zeta), \psi_{2}(\zeta), \psi_{3}(\zeta)\right]^{T}$, where $\psi_{i}(\zeta)$ are the eigenfunctions, and $\lambda$ is the system eigenvalues.

Lemma 1. The eigenvalues of the system are given as the solution of the following non-linear equation:

$$
\begin{gathered}
c(\lambda)=q_{1}\left[M_{31}(1, \lambda)-\rho_{1} M_{11}(1, \lambda)-\rho_{2} M_{21}(1, \lambda)\right] \\
+q_{2}\left[M_{32}(1, \lambda)-\rho_{1} M_{12}(1, \lambda)-\rho_{2} M_{22}(1, \lambda)\right]+\left[M_{33}(1, \lambda)-\rho_{1} M_{13}(1, \lambda)-\rho_{2} M_{23}(1, \lambda)\right]=0
\end{gathered}
$$

where $M_{i j}$ are the elements of the exponential matrix given by $\mathrm{e}^{V^{-1}\left(A^{*}-\lambda I\right) \zeta}$, where $V$ is a matrix with the system velocities and $A^{*}$ is the matrix with the in-domain coupling coefficients.

Proof. The operator $A$ can be written as $A=-V \partial_{\zeta}+A^{*}$, where $V$ is a matrix with the system velocities and $A^{*}$ is a matrix with the in-domain coupling coefficients. For the particular system considered (given in Equation (2)):

$$
V=\left[\begin{array}{ccc}
-\mu & 0 & 0 \\
0 & \gamma_{1} & 0 \\
0 & 0 & \gamma_{2}
\end{array}\right] ; A^{*}=\left[\begin{array}{ccc}
\alpha_{1} & \beta_{1} & \beta_{2} \\
\alpha_{1} & \sigma_{11} & \sigma_{12} \\
\alpha_{1} & \sigma_{21} & \sigma_{22}
\end{array}\right]
$$

Therefore, the eigenvalue problem can be written as:

$$
-V \partial_{\zeta} \psi(\zeta)+A^{*} \psi(\zeta)=\lambda \psi(\zeta) \rightarrow \partial_{\zeta} \psi(\zeta)=V^{-1}\left(A^{*}-\lambda I\right) \psi(\zeta)
$$

which has the following general solution:

$$
\psi(\zeta)=\mathrm{e}^{V^{-1}\left(A^{*}-\lambda I\right) \zeta} \psi(\zeta=0)
$$


with:

$$
\mathrm{e}^{V^{-1}\left(A^{*}-\lambda I\right) \zeta}=\left[\begin{array}{lll}
M_{11}(\zeta, \lambda) & M_{12}(\zeta, \lambda) & M_{13}(\zeta, \lambda) \\
M_{21}(\zeta, \lambda) & M_{22}(\zeta, \lambda) & M_{23}(\zeta, \lambda) \\
M_{31}(\zeta, \lambda) & M_{32}(\zeta, \lambda) & M_{33}(\zeta, \lambda)
\end{array}\right]
$$

Finally, applying the boundary conditions of Equations (3)-(14) with the definition given in Equation (15), the non-linear equation shown in Equation (11) is obtained.

In this contribution, the system parameters as shown in Table 1 are considered. For these values, the eigenvalue distribution is shown in Figure 2. With the values considered, it is possible to conclude that the steady-state considered generates an unstable linearized system. Furthermore, it has an infinity number of unstable eigenvalues, which would not be easily stabilized with techniques generally used for linear finite systems, such as pole-placement.

Table 1. Values of the parameters considered.

\begin{tabular}{cccc}
\hline Parameter & Value & Parameter & Value \\
\hline$q_{1}=q_{2}$ & 1 & $\rho_{1}=\rho_{2}$ & 0.5 \\
\hline$\sigma_{11}=\sigma_{21}=\beta_{1}$ & 0.2 & $\sigma_{12}=\sigma_{22}=\beta_{2}$ & 0.05 \\
\hline$\alpha_{1}$ & 0.1 & $\mu$ & 2 \\
\hline$\gamma_{1}$ & 0.5 & $\gamma_{2}$ & 1 \\
\hline
\end{tabular}

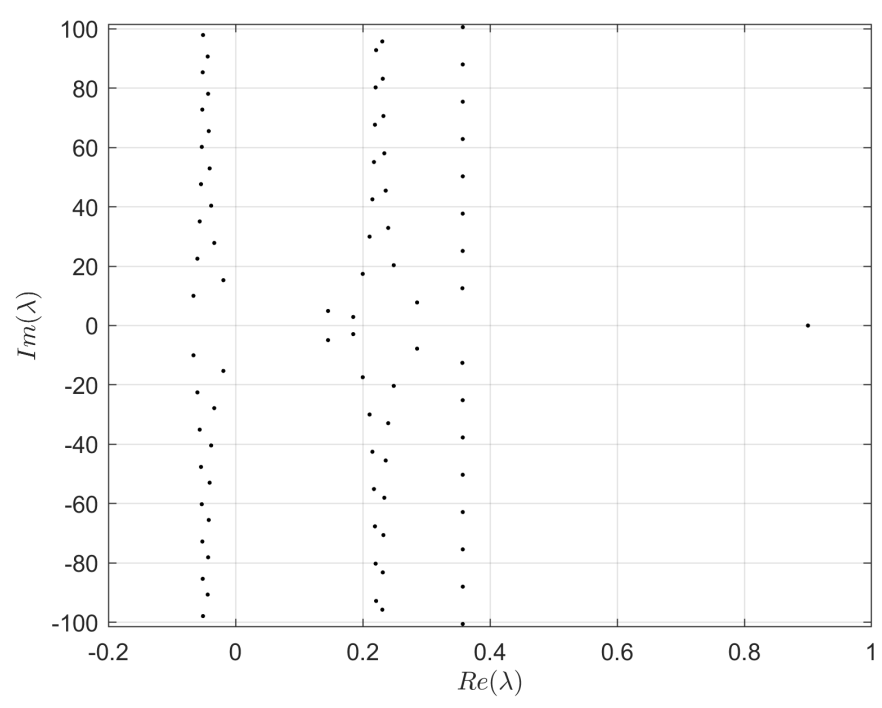

Figure 2. System eigenvalue distribution for the parameters given in Table 1.

\subsubsection{Resolvent and Transfer Function}

In this section, the system's resolvent and transfer function are derived, as they will be used in the following sections. The resolvent is used to generate the discrete representation of the system, shown in Section 4.1. The transfer function and the resolvent are also necessary to solve the Sylvester equations related to the output regulation problem, defined in Section 3.2.

Lemma 2. The system resolvent is given by:

$$
X(\zeta, s)=\underbrace{\mathrm{e}^{V^{-1}\left(A^{*}-s I\right) \zeta}\left\{\left[\begin{array}{c}
q_{1} \\
q_{2} \\
1
\end{array}\right] \frac{1}{c(s)} \int_{0}^{1}\left[g_{1}(1-\eta, s) g_{2}(1-\eta, s) g_{3}(1-\eta, s)\right] \bar{x}_{0}(\eta) d \eta+\int_{0}^{\zeta} \mathrm{e}^{-V^{-1}\left(A^{*}-s I\right) \eta} V^{-1} \bar{x}_{0}(\eta)\right\}}_{(s I-A)^{-1} \bar{x}_{0}}
$$


with $g_{i}(1-\eta, s)=\rho_{1} M_{1, i}(1-\eta, s)+\rho_{2} M_{2, i}(1-\eta, s)-M_{3, i}(1-\eta, s)$, with $M_{j, i}$ defined as in Equation (14) and $c(s)$ is the same function given in Equation (11) (as expected, if $\left.s=\lambda,(s I-A)^{-1} \nexists\right) .(s I-A)^{-1} B$ and the system transfer function are:

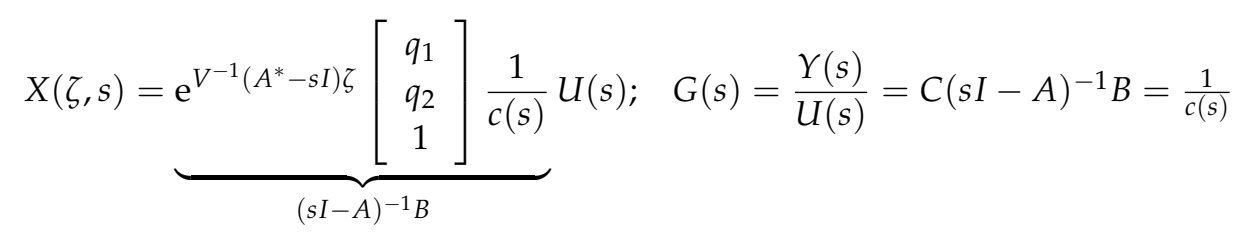

Proof. By applying Laplace Transform in the system defined by Equation (4), the following system is obtained:

$$
\left\{\begin{array}{l}
X(s)-x_{0}=A X(s)+B U(s) \\
Y(s)=C X(s)
\end{array}\right.
$$

Using the fact that $A(\cdot)=-V \partial_{\zeta}(\cdot)+A^{*}(\cdot)$ gives the general solution as:

$$
X(\zeta, s)=\mathrm{e}^{V^{-1}\left(A^{*}-\lambda s\right) \zeta} X(s, \zeta=0)+\int_{0}^{\zeta} V^{-1} \mathrm{e}^{V^{-1}\left(A^{*}-\lambda s\right)(\zeta-\eta)} x_{0}(\eta) d \eta
$$

By applying the boundary conditions shown in Equation (3), the general solution can be written as $X(\zeta, s)=(s I-A)^{-1} x_{0}+(s I-A)^{-1} B U(s)$ where the operator $(s I-A)^{-1}$ and the function $(s I-A)^{-1} B$ are the ones shown in Equations (16) and (17), respectively.

\section{Continuous Time Regulator Design}

First, the regulator in the continuous time setting is considered, as represented in Figure 3. It is necessary to find a control law that guarantees the closed-loop stability and proper output tracking using only the measured output information provided by the plant.

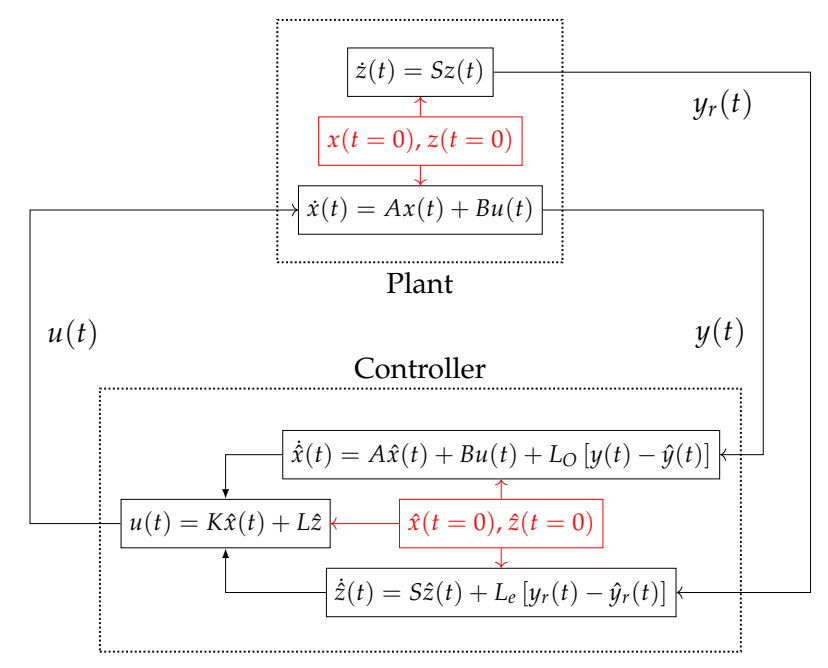

Figure 3. Continuous closed-loop representation.

\subsection{System Stabilization}

The first part of the controller is designed to guarantee the closed-loop stability of the system given by Equation (2) or, in a general form, by Equation (4), such that:

$$
u(t)=K x(t)+r(t)
$$

Which leads to a stable closed-loop system: 


$$
\begin{aligned}
& \dot{x}(t)=(A+B K) x(t)+B r(t)=\bar{A} x(t)+B r(t) \\
& y(t)=C x(t)
\end{aligned}
$$

Thus, it is desired to find a proper operator $K(\cdot)$ that achieves closed-loop stabilization ( $\bar{A}=A+B K$ is stable). To obtain closed-loop stability for the PDE system, backstepping is applied, as it was developed in [13] for continuous-time setting and it is shown in Appendix A. Although other methods could be applied to ensure the closed-loop stability [10-12], the backstepping methodology has been used for boundary control, as it maps the closed-loop system to a stable desired target system. Specifically for systems of hyperbolic PDEs, finite-time stabilization of the closed-loop system can be achieved by choosing a target system with this type of stability [18]. The following control law is able to map the closed-loop system into the stable target system (given by Equation (A4)):

$$
\begin{gathered}
u(t)=K[x(t)]+r(t)=-\rho_{1} x_{1}(t, \zeta=1)-\rho_{2} x_{2}(t, \zeta=1) \\
+\mathrm{e}^{-\frac{\alpha_{1}}{\mu}} \int_{0}^{1}\left[K_{1}(1, \eta) \mathrm{e}^{\frac{\alpha_{1}}{\mu} \eta} v(\eta, t)+K_{2}(1, \eta) x_{1}(\eta, t)+K_{3}(1, \eta) x_{2}(\eta, t)\right] d \eta+r(t)
\end{gathered}
$$

where the integral kernels $\left(K_{i}(\zeta, \eta)\right)$ need to satisfy the system of hyperbolic PDEs given by Equation (A3).

Remark 2. The target system shown in Equation (A4) can be represented in a general form as:

$$
\begin{aligned}
& \dot{\tilde{x}}(t)=\tilde{A} \tilde{x}(t)+\tilde{B} r^{\prime}(t) \\
& y(t)=\tilde{C} \tilde{x}(t)
\end{aligned}
$$

If the transformation $T$ shown in Equation ( $A 2)$ exists, then the closed loop system $(A+B K)$ can be transformed in the target system. Therefore, the transformation is such that $\tilde{x}(t)=T x(t)$ and $x(t)=T^{-1} \tilde{x}(t)$, and, finally, $T(A+B K) T^{-1}=\tilde{A}$. From linear algebra, the following properties of the transformation of a linear system are guaranteed:

$$
\begin{gathered}
\sigma(A+B K)=\sigma(\tilde{A}) \\
{[s I-(A+B K)]^{-1}(\cdot)=T^{-1}\left[(s I-\tilde{A})^{-1}\right] T(\cdot)} \\
{[s I-(A+B K)]^{-1} B=T^{-1}\left[(s I-\tilde{A})^{-1}\right] T B=T^{-1}\left[(s I-\tilde{A})^{-1}\right] \tilde{B}} \\
G_{C L}(s)=C[s I-(A+B K)]^{-1} B=C T^{-1}\left[(s I-\tilde{A})^{-1}\right] T B=\tilde{C}\left[(s I-\tilde{A})^{-1}\right] \tilde{B}=\tilde{G}(s)
\end{gathered}
$$

Therefore, the closed-loop will be stable if the target system is stable (and the transformation $T$ and its inverse exist).

Lemma 3. The closed-loop system will reach the origin in a finite-time of $t=t_{s}=\phi_{1}+\phi_{2}$, with $\phi_{1}=\frac{1}{\mu}$ and $\phi_{2}=\max \left\{\frac{1}{\gamma_{1}}, \frac{1}{\gamma_{2}}\right\}$ and $r(t)=0$.

Proof. From the linear properties shown in Remark 2, it is important to notice that the closed-loop dynamics will not necessarily be exactly the same as the target system. But, due to the structure of the target system shown in Equation (A4), it is easy to see that if $r(t)=0$, then $r^{\prime}(t)=0$ and $W(\zeta, t>$ $\left.\phi_{1}\right)=0, \forall \zeta \in[0,1]$, with $\phi_{1}=\frac{1}{\mu}$. This also implies that $X_{1}\left(0, t>\phi_{1}\right)=X_{2}\left(0, t>\phi_{1}\right)=0$ due to the boundary conditions, which finally leads to $X_{1}\left(\zeta, t>\phi_{1}+\phi_{2}\right)=X_{2}\left(\zeta, t>\phi_{1}+\phi_{2}\right)=0, \forall \zeta \in[0,1]$, where $\phi_{2}=\max \left\{\frac{1}{\gamma_{1}}, \frac{1}{\gamma_{2}}\right\}$. Therefore, the target system will reach steady-state $(\tilde{x}(\zeta, t)=0)$ in a finite-time of $t=t_{s}=\phi_{1}+\phi_{2}$. Due the transformation $T$, the closed-loop states will be $x(t)=T^{-1} \tilde{x}(t)$ ), thus $x(\zeta, t)=0, \forall \zeta \in[0,1]$ for $t \geq t_{s}$. 
Thus, the control law given in Equation (22) will stabilize the PDE system in a finite-time $t_{s}$. With the system stabilized, it is expected that system's properties (such as the resolvent, and consequently, the transfer function) will change in the closed-loop.

Lemma 4. The closed-loop resolvent is given by:

$$
\begin{gathered}
(s I-\bar{A})^{-1}(\cdot)=\left\{(s I-A)^{-1}+\right. \\
\left.(s I-A)^{-1} B\left[I-K(s I-A)^{-1} B\right]^{-1} K(s I-A)^{-1}\right\}(\cdot)
\end{gathered}
$$

which leads to the closed-loop transfer function as:

$$
\begin{gathered}
\bar{G}(s)=C(s I-\bar{A})^{-1} B= \\
G(s)\left\{I+\left[I-K(s I-A)^{-1} B\right]^{-1} K(s I-A)^{-1} B\right\}
\end{gathered}
$$

Proof. The closed-loop resolvent is obtained as follows:

$$
(s I-\bar{A})^{-1}(\cdot)=(s I-A-B K)^{-1}(\cdot)=(s I-A)^{-1}\left[I-B K(s I-A)^{-1}\right]^{-1}
$$

And applying Woodbury identity to the term in brackets gives the resolvent shown in Equation (25). By using the definition of the open-loop transfer function $\left(G(s)=C(s I-A)^{-1} B\right)$, Equation (26) is easily obtained.

Therefore, by knowing $(s-A)^{-1} B$ and the feedback control gain $K$, it is possible to easily calculate the closed-loop properties. Although Equations (25) and (26) might seem to increase the complexity on finding the closed-loop properties, control laws as given in Equation (22) will result in a integral equation if one tries to calculate these properties directly.

\subsection{Output Regulation}

The last step in the design of the regulator in the continuous-time setting is finding the feedforward gain $L$ that achieves proper tracking of the signal generated by the exosystem states $(z(t))$ given by Equations (5) and (6). The control law obtained after taking the feedforward gain $L$ into account is given by Equation (10)

$$
u(t)=K x(t)+L z(t)
$$

Lemma 5. The proper output regulation can be easily achieved by solving the following Sylvester equation:

$$
\begin{aligned}
& \Pi S=\bar{A} \Pi+B L=(A+B K) \Pi+B L \\
& C \Pi=Q
\end{aligned}
$$

Proof. First, the error $e_{r}(t)=x(t)-\Pi z(t)$ is defined, and, considering that $u(t)=K[x(t)]+r(t)$, taking the time derivative gives :

$$
\dot{e}_{r}(t)=\dot{x}(t)-\Pi \dot{z}(t)=(A+B K) x(t)-\Pi S z(t)+B r(t)
$$

If we consider that there is a feedforward gain $L$, such that $r(t)=L z(t)$, and by adding and subtracting $(A+B K) \Pi z(t)$ :

$$
\dot{e}_{r}(t)=(A+B K)[x(t)-\Pi z(t)]+[(A+B K) \Pi z(t)-\Pi S z(t)+B L z(t)]
$$


And by making the term in the second bracket equal zero:

$$
\dot{e}_{r}(t)=(A+B K) e_{r}(t)
$$

which generates a stable system if Equation (29) holds. Notice that the tracking error $e(t)$, defined in Equation (9), will be:

$$
C e_{r}(t)=C x(t)+C \Pi z(t)=y(t)-y_{r}(t)+[C \Pi z(t)-Q z(t)]
$$

Therefore, if $C \Pi=Q$, then $C e_{r}(t)=e(t)$, and, as $e_{r}(t)$ is stable, $e(t)$ will decrease and proper tracking is achieved.

Remark 3. From Remark 2 and Lemma 3, it is possible to say that $e_{r}(t)$ is going to reach the origin at a finite time $t_{s}$. Thus, $y(t)=y_{r}(t)$ for $t>t_{s}$, as $e(t)=C e_{r}(t)=0$ for $t>t_{s}$. Also, as $e_{r}(t)=0$ for $t>t_{s}$, $x(t)=\Pi z(t)$ and $\Pi$ can be recognized as a mapping between the system states and the exosystem states when proper tracking is achieved.

Remark 4. Due to the exosystem structure defined in Assumption 1, the Sylvester equation shown in Equation (29) can be separated in two different sets of equations and solved separately.

Lemma 6. Considering the same number of inputs as of outputs, the solution for the first set of Sylvester equations shown in Remark 4 will be:

$$
\begin{gathered}
L_{1, i}=\left[\bar{G}_{1}(0)\right]^{-1}\left[Q_{1, i}+\sum_{j=1}^{i-1}(-1)^{i-(j-1)} \bar{G}_{i-(j-1)}(0) L_{1, j}\right] \\
\Pi_{1, i}=\sum_{j=1}^{i}(-1)^{i-j}\left[(0-\bar{A})^{-1}\right]^{i-(j-1)} B L_{1, j}
\end{gathered}
$$

for $i=1, \ldots, n_{1}$, where $L_{1}=\left[L_{1,1}, \ldots, L_{1, n_{1}}\right], \Pi_{1}=\left[\Pi_{1,1}, \ldots, \Pi_{1, n_{1}}\right]$ and $\bar{G}_{k}(0)=C\left[(0-\bar{A})^{-1}\right]^{k} B$, such that $\bar{G}_{1}(0)=C\left[(0-\bar{A})^{-1}\right] B=\bar{G}(0)$. And for the second set of equations, the solution will be:

$$
\begin{gathered}
L_{2,2 i-1}=\operatorname{Re}\left[\bar{G}\left(f_{i} \mathbf{j}\right)^{-1}\right] Q_{2,2 i-1}-\operatorname{Im}\left[\bar{G}\left(f_{i} \mathbf{j}\right)^{-1}\right] Q_{2,2 i} \\
L_{2,2 i}=\operatorname{Re}\left[\bar{G}\left(f_{i} \mathbf{j}\right)^{-1}\right] Q_{2,2 i}+\operatorname{Im}\left[\bar{G}\left(f_{i} \mathbf{j}\right)^{-1}\right] Q_{2,2 i-1} \\
\Pi_{2,2 i-1}(\zeta)=\operatorname{Re}\left[\left(f_{i} \mathbf{j} I-\bar{A}\right)^{-1} B\right] L_{2,2 i-1}-\operatorname{Im}\left[\left(f_{i} \mathbf{j} I-\bar{A}\right)^{-1} B\right] L_{2,2 i} \\
\Pi_{2,2 i}(\zeta)=\operatorname{Re}\left[\left(f_{i} \mathbf{j} I-\bar{A}\right)^{-1} B\right] L_{2,2 i}+\operatorname{Im}\left[\left(f_{i} \mathbf{j} I-\bar{A}\right)^{-1} B\right] L_{2,2 i-1}
\end{gathered}
$$

for $i=1, \ldots, n_{2} / 2$, where $L_{2}=\left[L_{2,1}, \ldots, L_{2, n_{2}}\right]$ and $\Pi_{2}=\left[\Pi_{2,1}, \ldots, \Pi_{2, n_{2}}\right]$.

Proof. For the first set of Sylvester equation it is easy to see that due to the structure of $J_{1}$ considered in Assumption 1, the following relation must hold:

$$
\Pi_{1, i}=\bar{A} \Pi_{1, i+1}+B L_{1, i+1}
$$

for $i=1, \ldots, n_{1}-1$ and with $0=\bar{A} \Pi_{1,1}+B L_{1,1}$. Applying $(0-\bar{A})^{-1}$ on both sides and isolating $\Pi$ leads to:

$$
\Pi_{1, i+1}=-(0-\bar{A})^{-1} \Pi_{1, i}+(0-\bar{A})^{-1} B L_{1, i+1}
$$

with $\Pi_{1,1}=(0-\bar{A})^{-1} B L_{1,1}$, which recursively leads to $\Pi_{1, i}$ in Equation (34). Applying $C$ in both sides of this last equation, taking into account that $C \Pi_{1, i}=Q_{1, i}$ and isolating for $L_{1, i}$ leads to the result shown in (34). 
For the second set of Sylvester equations, first, both sides of the equation are multiplied by the eigenvector of $J_{2}$, and taking into account that $J_{2} \phi_{J_{2}}=\lambda_{J_{2}} \phi_{J_{2}}$ leads to:

$$
\Pi_{2} J_{2} \phi_{J_{2}}=\Pi_{2} \lambda_{J_{2}} \phi_{J_{2}}=\bar{A} \Pi_{2} \phi_{J_{2}}+B L_{2} \phi_{J_{2}}
$$

From Remark 1, the eigenvectors of $J_{2}$ are all null except at the corresponding row of $\pm f_{i} \mathbf{j}$, with $i=1, \ldots, n_{2} / 2$. Therefore:

$$
\begin{aligned}
\Pi_{2} \phi_{J_{2}} & =\left(\lambda_{J_{2}} I-\bar{A}\right)^{-1} B L_{2} \phi_{J_{2}} \\
{\left[\Pi_{2,2 i-1} \Pi_{2,2 i}\right][1 \mathbf{j}]^{T} } & =\left(f_{i} \mathbf{j} I-\bar{A}\right)^{-1} B\left[L_{2,2 i-1} L_{2,2 i}\right][1 \mathbf{j}]^{T}
\end{aligned}
$$

and the equivalence of the complex numbers from both sides of the equations gives the solution for $\Pi_{2,2 i-1}$ and $\Pi_{2,2 i}$. Applying $C$ in both sides, knowing that $C \Pi_{2, i}=Q_{2, i}$ gives the results shown in Equation (35).

Remark 5. From the solution shown in Lemma 6, it is possible to conclude that the solutions of the Sylvester equations $\Pi$ exist if $\sigma(\bar{A}) \neq \sigma(S)=\sigma\left(J_{1}\right) \cup \sigma\left(J_{2}\right)$, such that $\bar{G}\left(\lambda_{S}\right) \neq \infty$. In a similar way, for the feedforward gains $L$ to exist the eigenvalues $S$ must not be a zero of the transfer function, as the inverse of the transfer function must exist (which means that $\bar{G}\left(\lambda_{S}\right) \neq 0$, such that L exist in Equations (34) and (35)).

For the exosystem shown in Equation (8), the solution of the Sylvester equations will be $L=$ $\left[L_{1,1} L_{1,2} L_{2,1} L_{2,2}\right]$ and $\Pi=\left[\Pi_{1,1} \Pi_{1,2} \Pi_{2,1} \Pi_{2,2}\right]$, given by:

$$
\begin{array}{cc} 
& L_{1,1}=\bar{G}(0)^{-1} Q_{1,1} \\
L_{1,2}=\bar{G}(0)^{-1}\left[Q_{1,2}+\bar{G}_{2}(0) L_{1,1}\right] & \Pi_{1,1}(\zeta)=(0-\bar{A})^{-1} B L_{1,1} \\
& \Pi_{1,2}=(0-\bar{A})^{-1}(0-\bar{A})^{-1} B L_{1,1}+(0-\bar{A})^{-1} B L_{1,2}
\end{array}
$$

and:

$$
\begin{aligned}
& L_{2,1}=\operatorname{Re}\left[\bar{G}(f \mathbf{j})^{-1}\right] Q_{2,1}-\operatorname{Im}\left[\bar{G}(f \mathbf{j})^{-1}\right] Q_{2,2} \quad \Pi_{2,1}(\zeta)=\operatorname{Re}\left[(f \mathbf{j} I-\bar{A})^{-1} B\right] L_{2,1}-\operatorname{Im}\left[(f \mathbf{j} I-\bar{A})^{-1} B\right] L_{2,2} \\
& L_{2,2}=\operatorname{Re}\left[\bar{G}(f \mathbf{j})^{-1}\right] Q_{2,2}+\operatorname{Im}\left[\bar{G}\left(f \mathbf{j}^{-1}\right] Q_{2,1} \quad \Pi_{2,2}(\zeta)=\operatorname{Re}\left[(f \mathbf{j} I-\bar{A})^{-1} B\right] L_{2,2}+\operatorname{Im}\left[(f \mathbf{j} I-\bar{A})^{-1} B\right] L_{2,1}\right.
\end{aligned}
$$

\subsection{System Observer Design}

The control law defined in Equation (22) needs a fullstate feedback measurement across the whole domain. As this is generally not practical or viable, in this section the observer design for thesystem shown in Equations (2) and (3) is considered. The observer dynamics will be given by the following set of PDE's:

$$
\left\{\begin{array}{l}
\frac{\partial \hat{v}}{\partial t}=\mu \frac{\partial \hat{v}}{\partial \zeta}+\alpha_{1} \hat{v}+\beta_{1} \hat{x}_{1}+\beta_{2} \hat{x}_{2}+\mathrm{e}^{-\frac{\alpha_{1}}{\mu} \zeta} l_{1}(\zeta)\left[y_{m}(t)-\hat{v}(\zeta=0)\right] \\
\frac{\partial \hat{x}_{1}}{\partial t}=-\gamma_{1} \frac{\partial \hat{x}_{1}}{\partial \zeta}+\alpha_{1} \hat{v}+\sigma_{11} \hat{x}_{1}+\sigma_{12} \hat{x}_{2}+l_{2}(\zeta)\left[y_{m}(t)-\hat{v}(\zeta=0)\right] \\
\frac{\partial \hat{x}_{2}}{\partial t}=-\gamma_{2} \frac{\partial \hat{x}_{2}}{\partial \zeta}+\alpha_{1} \hat{v}+\sigma_{21} \hat{x}_{1}+\sigma_{22} \hat{x}_{2}+l_{3}(\zeta)\left[y_{m}(t)-\hat{v}(\zeta=0)\right] \\
\hat{v}(1, t)=\rho_{1} \hat{x}_{1}(1, t)+\rho_{2} \hat{x}_{2}(1, t)+u(t) \\
\hat{x}_{1}(0, t)=q_{1} y_{m}(t) \\
\hat{x}_{2}(0, t)=q_{2} y_{m}(t)
\end{array}\right.
$$

where $\hat{x}(\zeta, t)=\left[\hat{v} \hat{x}_{1} \hat{x}_{2}\right]^{T} \in L^{2}(0,1)$ are the reconstructed states and $l_{i}(\zeta)$ are the in-domain observer gains. Similarly to the system stabilization shown in Section 3.1 and Appendix A, the backstepping methodology is used once again to find the appropriate gains for the observer. Other techniques could be used, but, once again, the backstepping methodology can be used to ensure finite-time 
convergence of the observer states to the system states. The derivation of the observer gains are shown in Appendix B.

The observer dynamics can be written in a general state-space representation as:

$$
\begin{aligned}
& \dot{\hat{x}}(t)=\hat{A} \hat{x}(t)+L_{o, 1}\left[y(t)-C_{m} \hat{x}(t)\right]+L_{o, 2}[y(t)]+B u(t)= \\
& \dot{\hat{x}}(t)=\hat{A} \hat{x}(t)+L_{o, 1} C_{m}[\bar{x}(t)-\hat{x}(t)]+L_{o, 2} C_{m}[\bar{x}(t)]+B u(t)
\end{aligned}
$$

where $L_{o, 1}$ is related to the in-domain observer gains and $L_{o, 2}$ is related to the boundary observer gains shown in Equation (A6). Although $\hat{A}$ has the same in-domain operators as $A$, it has different boundary conditions, such that $A=\hat{A}+L_{0,2} C_{m}$.

Lemma 7. The system observer error will reach the origin in a finite-time of $t=t_{0}=\phi_{2}+\phi_{1}$, with $\phi_{2}=\max \left\{\frac{1}{\gamma_{1}}, \frac{1}{\gamma_{2}}\right\}$ and $\phi_{1}=\frac{1}{\mu}$

Proof. Due to the structure of the target system shown in Equation (A9), it is easy to see that $\tilde{e}_{1}(\zeta=$ $\left.0, t>\phi_{2}\right)=\tilde{e}_{2}\left(\zeta=0, t>\phi_{2}\right)=0, \forall \zeta \in[0,1]$, with $\phi_{2}=\max \left\{\frac{1}{\gamma_{1}}, \frac{1}{\gamma_{2}}\right\}$. This also implies that $\tilde{e}_{3}\left(\zeta=0, t>\phi_{2}\right)=0$ due to the boundary condition. Finally, $\tilde{e}_{3}\left(\zeta, t>\phi_{1}+\phi_{2}\right)=0, \forall \zeta \in[0,1]$, where $\phi_{1}=\frac{1}{\mu}$. Therefore, the observer error target system will reach the origin $(\tilde{e}(\zeta, t)=0)$ in a finite-time of $t=t_{o}=\phi_{2}+\phi_{1}$. As backstepping is just a linear transformation of the observer error, such that the original observer error is related to the target system by $\hat{e}=T^{-1} \tilde{e}(t), \hat{e}(t)=0, \forall \zeta \in[0,1]$ for $t \geq t_{0}$.

\subsection{Exosystem Observer}

If the exosystem dynamics is known, but its states are not available, it is possible to reconstruct the states using the reference signal $y_{r}(t)$. As the exosystem considered here is a finite system (as shown in Equations (5) and (6)), the following observer is considered:

$$
\dot{\hat{z}}(t)=S \hat{z}(t)+L_{e}\left[y_{r}(t)-Q \hat{z}(t)\right]=S \hat{z}(t)+L_{e} Q[z(t)-\hat{z}(t)]
$$

where $z$ is the estimated exosystem state and $L_{e}$ is the observer gain to be defined. The exosystem observer error is given by $\hat{e}_{e}(t)=z(t)-\hat{z}(t)$ and its dynamics will be:

$$
\dot{\hat{e}}_{e}(t)=\left(S-L_{e} Q\right) \hat{e}_{e}(t)
$$

If $(S, Q)$ is detectable, then it is possible to guarantee the reconstruction of the significant exosystem states and stabilization can be achieved by using pole-placement.

\section{Exosystem Finite-Time Observer}

The observer design from the previous section attains stabilization in a exponential matter. Here, the finite-time observer is considered to obtain a faster estimation of the exosystem observer. This kind of stabilization was shown in [24] and used in [25]. For this design, two exosystem observers $\left(\hat{z}_{1}\right.$ and $\left.\hat{z}_{2}\right)$ with different observer gains $\left(L_{e, 1}\right.$ and $\left.L_{e, 2}\right)$ are considered and the redundant information from this two observers can be used in combination with previous information to get the estimation to converge in a finite-time. This observer equations are given as:

$$
\hat{z}_{F}(t)=\left[\begin{array}{ll}
I & 0
\end{array}\right]\left[\begin{array}{ll}
I & \mathrm{e}^{\left(A-L_{e, 1} Q\right) D_{F}} \\
I & \mathrm{e}^{\left(A-L_{e, 2} Q\right) D_{F}}
\end{array}\right]^{-1}\left(\left[\begin{array}{l}
\hat{z}_{1}(t) \\
\hat{z}_{2}(t)
\end{array}\right]-\left[\begin{array}{l}
\mathrm{e}^{\left(A-L_{e, 1} Q\right) D_{F}} \hat{z}_{1}\left(t-D_{F}\right) \\
\mathrm{e}^{\left(A-L_{e, 2} Q\right) D_{F}} \hat{z}_{2}\left(t-D_{F}\right)
\end{array}\right]\right)
$$

where $\hat{z}_{F}(t)$ is the estimation given by the finite-time observer, $D_{F}$ is the time delay used, $\mathrm{e}^{\left(A-L_{e, i} Q\right) D_{F}}$ is the exponential matrix and $I$ is the identity matrix. With this observer design, $\hat{z}_{F}(t)$ is guaranteed to converge to $z(t)$ when $t>D_{F}$ as shown in [24]. 


\section{Discrete Time Regulator Design}

The discrete regulator design is considered in this section. It is necessary to find a control law for the discrete system that guarantees the closed-loop stability and proper output tracking. Figure 4 represents the closed-loop controller for the discrete setting. The closed-loop begins at the controller, where the initial condition for the system and exosystem observers $\left(\hat{x}_{k=-1}\right.$ and $\left.\hat{z}_{k=-1}\right)$ are used to calculate the first control action $u_{k=0}$. Then, the action is applied to the plant and the system measured output and the reference signal from the exosystem become available $\left(y_{k=0}\right.$ and $\left.y_{r, k=0}\right)$. These output are used to estimate the system and exosystem states in the observer $\left(\hat{x}_{k=0}\right.$ and $\left.\hat{z}_{r, k=0}\right)$. Finally, the estimates are used in the control law to calculate the next input $\left(u_{1}\right)$ and the process is repeated.

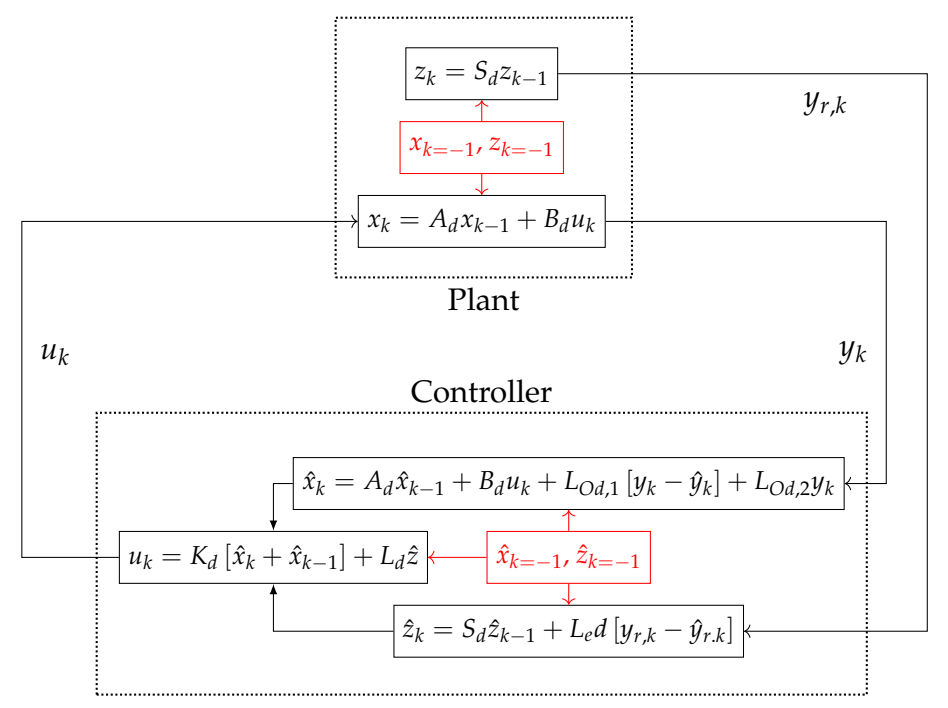

Figure 4. Discrete time closed-loop representation.

The control law used to ensure the system stability and proper signal tracking is similar to the regulator equation shown in Equation (10) for the continuous setting considering fullstate feedback:

$$
u_{k}=K_{d} x_{k-1}+L_{d} z_{k-1}
$$

where the first part of the right side of the control law represents the feedback control used to guarantee the system stability. The second part is responsible for the output tracking and $L_{d}$ is a matrix that needs to be found to ensure proper output tracking.

The discrete output tracking error can be defined as:

$$
e_{k}=y_{k}-y_{r, k}
$$

Similar to the controller in the continuous time setting, the discrete regulator should guarantee:

- The closed-loop system is stable;

- For the closed-loop system, the tracking error $e_{k}(k \rightarrow \infty)=0, \forall x_{k=0} \in X, z_{k=0} \in \mathbb{R}$;

Before addressing the discrete regulator design, first the proper discrete representation of the system is considered.

\subsection{Discrete Representation}

Given the system defined in Equation (4), one can apply a structure preserving time discretization of the dynamical system. By the application of Crank-Nicolson midpoint integration rule, and with 
the assumption of piecewise constant input within the sampled intervals, the Cayley-Tustin time discretization transformation is used. The obtained discrete system is represented as:

$$
\begin{gathered}
x_{k}=A_{d} x_{k-1}+B_{d} u_{k} \\
y_{k}=C_{d} x_{k-1}+D_{d} u_{k} \\
y_{m, k}=C_{m, d} x_{k-1}+D_{m, d} u_{k}
\end{gathered}
$$

where $\delta=2 / \Delta t, A_{d}, B_{d}, C_{d}, D_{d}, C_{m, d}$ and $D_{m, d}$ are the discrete time system operators and are given by:

$$
\left[\begin{array}{cc}
A_{d}(\cdot) & B_{d} \\
C_{d}(\cdot) & D_{d} \\
C_{m, d}(\cdot) & D_{m, d}
\end{array}\right]=\left[\begin{array}{cc}
-I+2 \delta(\delta I-A)^{-1}(\cdot) & \sqrt{2 \delta}(\delta I-A)^{-1} B \\
\sqrt{2 \delta} C(\delta I-A)^{-1}(\cdot) & C(\delta I-A)^{-1} B \\
\sqrt{2 \delta} C_{m}(\delta I-A)^{-1}(\cdot) & C_{m}(\delta I-A)^{-1} B
\end{array}\right]
$$

and $(\delta I-A)^{-1}=R(\delta, A)$ is defined as the resolvent operator of the operator $A$, which can be found in Equation (16), $(\delta I-A)^{-1} B$ and $G(\delta)=C(\delta I-A)^{-1} B$ were defined in Equation (17) for the specific system considered.

The operators given by Equation (50) are all compact and well-defined and the issue of boundary (point) actuation or/and observation does not induce mathematical difficulties which are associated with the continuous counterparts, usually leading to unboundness of the boundary (point) actuation and/or observation.

Assumption 2. A small enough value of $\Delta t$ is used such that the discrete time representation of the system shown in Equation (49) is a good approximation of the open-loop system internal dynamics and input/output relation.

Assumption 2 presumes there is a large enough $\delta$ (small enough $\Delta t$ ) such that the discretization can be applied to unstable systems as well [26] and is necessary for the development of the discrete regulator in the following sessions. For an unstable open-loop system, the following lemma gives an interval for which the discrete representation might be a good approximation of the system:

Lemma 8. In the case of an unstable system, the discrete system $\left(A_{d}, B_{d}, C_{d}, D_{d}\right)$ will not be a good discrete approximation of the system $(A, B, C, D)$ if $\Delta t>\frac{2}{s(A)}$, where $s(A)=\sup \{\operatorname{Re} z: z \in \sigma(A)\}$.

Proof. For a stable system, $s(A)<0$ (all eigenvalues are negative), thus, as $\delta=\frac{2}{\Delta t}$ and $\Delta t>0$, $(\delta I-A)^{-1}$ exists for any value of $\Delta t$. For an unstable system, $s(A)>0$, there will be at least one unstable eigenvalue with a positive real part. If this eigenvalue is real, $\delta \rightarrow s(A)$ and $(\delta I-A)^{-1} \rightarrow \infty$, if $\Delta t \rightarrow \frac{2}{s(A)}$. As the discrete operators shown in Equation (49) depend on the resolvent, $\Delta t \geq \frac{2}{s(A)}$ will not result in a good discrete approximation. If the eigenvalue is complex, the Spectral Mapping Theorem can give an insight on the behavior of the discrete eigenvalue. If there exists a exact discrete representation of $A$, it's largest eigenvalue will be $\lambda_{d, E}=\mathrm{e}^{\frac{2}{\delta} \lambda_{s}}$, such that $\lambda_{s}=a+b \mathbf{j}$ and $\operatorname{Re} \lambda_{s}=a=$ $s(A)$. For the Cayley-Tustin transformation, this relation is given by $\lambda_{d, C T}=\frac{\delta+\lambda_{s}}{\delta-\lambda_{s}}$. If we calculate the modulus of these eigenvalues, we get:

$$
\left\|\lambda_{d, E}\right\|=\mathrm{e}^{\frac{2 a}{\delta}} ;\left\|\lambda_{d, C T}\right\|=\sqrt{1+\frac{4 a \delta+\left\|\lambda_{S}\right\|^{2}}{\delta^{2}-2 a \delta+\left\|\lambda_{s}\right\|^{2}}}
$$
yields:

As expected, if $\Delta t \rightarrow 0, \delta \rightarrow \infty$ and $\left\|\lambda_{d, C T}\right\| \rightarrow\left\|\lambda_{d, E}\right\|$. Taking the derivative with respect to $\delta$

$$
\frac{\partial\left\|\lambda_{d, E}\right\|}{\partial \delta}=\frac{-2 a}{\delta^{2}} \mathrm{e}^{\frac{2 a}{\delta}} ; \frac{\partial\left\|\lambda_{d, C T}\right\|}{\partial \delta}=\frac{2 a\left(\left\|\lambda_{s}\right\|^{2}-\delta^{2}\right)}{\left(\left\|\lambda_{s}\right\|^{2}-2 a \delta+\delta^{2}\right)^{2}|| \lambda_{d, C T} \|}
$$


For the exact representation, the value of the discrete eigenvalues will decrease as $\delta$ increases (i.e., $\Delta t$ decreases). For the Cayley-Tustin transformation it is possible to see that the function will have an turning point at $(\delta)_{\text {Turn }}=\left\|\lambda_{s}\right\|$ or $(\Delta t)_{\text {Turn }}=\frac{2}{\left\|\lambda_{s}\right\|}$. If $\delta=\frac{2}{\Delta t}=s(A) \leq\left\|\lambda_{s}\right\|$, then $\Delta t>(\Delta t)_{\text {Turn }}$ and the functions have different responses, i.e., $\| \lambda_{d, E}||$ is increasing as $\Delta t$ increases, but $\left\|\lambda_{d, C T}\right\|$ will decrease past the turning point. Thus, $\Delta t \geq \frac{2}{s(A)}$ will not generate a good discrete approximation of the system.

Similar to the controller design in the continuous time setting, first the stabilization of the system in the discrete time setting given by Equations (49) and (50) is addressed.

\subsection{Discrete System Stabilization}

The design of the discrete regulator is considered in this section. First, it is shown in Lemma 9 that the discrete system can be stabilized.

Lemma 9. If $K$ in the continuous time setting is able to stabilize the system, then there is a corresponding $K_{d}(\cdot)$ in the discrete time setting that ensures the discrete system stability with the control law:

$$
u_{k}=K_{d} x_{k-1}
$$

with:

$$
K_{d}=\sqrt{2 \delta}\left(I-K(\delta I-A)^{-1} B\right)^{-1} K(\delta I-A)^{-1}
$$

Proof. With the control law considered, the closed-loop discrete system is given by:

$$
x_{k}=\left(A_{d}+B_{d} K_{d}\right) x_{k-1}
$$

By using the definitions of the operators, the following relation can be achieved:

$$
\begin{gathered}
x_{k}=\left[-I+2 \delta(\delta I-A)^{-1}+2 \delta(\delta I-A)^{-1} B\left(I-K(\delta I-A)^{-1} B\right)^{-1} K(\delta I-A)^{-1}\right] x_{k-1}= \\
\left\{-I+2 \delta(\delta I-A)^{-1}\left[I-B K(\delta I-A)^{-1}\right]^{-1}\right\} x_{k-1}=\left[-I+2 \delta(\delta I-A-B K)^{-1}\right] x_{k-1}
\end{gathered}
$$

where the Woodbury identity was used to properly manipulate the expressions. The resulting operator is equivalent to the discrete operator obtained by applying the Cayley-Tustin time discretization in the closed-loop system obtained in Section 3.1. Using the Cayley-Tustin transformation, the closed-loop system can be represented in the discrete setting as:

$$
x_{k}=\bar{A}_{d} x_{k-1} \rightarrow \bar{A}_{d}=-I+2 \delta(\delta I-\bar{A})^{-1}=-I+2 \delta(\delta I-A-B K)^{-1}
$$

From $[27,28]$, it was shown that a stable continuous operator generates a stable discrete operator, thus, if $(A+B K)$ is stable, then the discrete operator $\bar{A}_{d}=-I+2 \delta(\delta I-A-B K)^{-1}$ is stable as well. Finally, if the control law proposed in Equation (53) with $K_{d}$ shown in Equation (54), is used, then the closed-loop discrete operator is equivalent to the operator shown above and the discrete closed-loop system is stable as well (i.e., $A_{d}+B_{d} K_{d}=\bar{A}_{d}$ ).

With the proposed control law, the discrete closed-loop system is given as:

$$
\begin{aligned}
& x_{k}=\bar{A}_{d} x_{k-1}+B_{d} r_{k} \\
& y_{k}=\bar{C}_{d} x_{k-1}+D_{d} r_{k}
\end{aligned}
$$


with:

$$
\left[\begin{array}{ll}
\bar{A}_{d} & B_{d} \\
\bar{C}_{d} & D_{d}
\end{array}\right]=\left[\begin{array}{ll}
A_{d}+B_{d} K_{d} & B_{d} \\
C_{d}+D_{d} K_{d} & D_{d}
\end{array}\right]=\left[\begin{array}{cc}
{\left[-I+2 \delta(\delta I-A-B K)^{-1}\right.} & B_{d} \\
\sqrt{2 \delta} C(I \delta-A-B K)^{-1} & D_{d}
\end{array}\right]
$$

\subsection{Discrete Output Regulation}

After achieving the system stabilization, it is possible to accomplish proper output tracking. First, the Cayley-Tustin time discretization is applied to the exosystem, such that the discrete time exosystem is given as:

$$
\begin{aligned}
z_{k} & =S_{d} z_{k-1} \\
y_{r, k} & =Q_{d} z_{k-1}
\end{aligned}
$$

with:

$$
S_{d}=-I+2 \delta(\delta-S)^{-1}=\left[\begin{array}{cccc}
1 & \frac{2}{\delta} & 0 & 0 \\
0 & 1 & 0 & 0 \\
0 & 0 & \frac{\delta^{2}-f^{2}}{\delta^{2}+f^{2}} & \frac{2 \delta f}{\delta^{2}+f^{2}} \\
0 & 0 & -\frac{2 \delta f}{\delta^{2}+f^{2}} & \frac{\delta^{2}-f^{2}}{\delta^{2}+f^{2}}
\end{array}\right] \quad Q_{d}=\sqrt{2 \delta} Q(\delta I-S)^{-1}
$$

Then, it is necessary to find the discrete feedforward gain $L_{d}$ that is able to guarantee the proper tracking of the reference signal $y_{r, k}$. This can be accomplished by solving the output regulator equations in the discrete-time setting.

\subsubsection{Discrete Regulator Equations}

In Section 3.2, the regulation equations were solved in the continuous setting. In this section, the output regulation equations for the discrete time setting are derived. The following Lemma summarizes the results for the output tracking if the Cayley-Tustin time discretization is applied.

Lemma 10. If the Cayley-Tustin time discretization is considered, then the solution of the regulation equation (Sylvester equations) in the discrete setting is the same as in the continuous setting, i.e., $\Pi_{d}=\Pi$, such that $x_{k}=\Pi_{d}(\zeta) z_{k}$ when proper tracking is achieved and it is the solution of the following Sylvester equations in the discrete-time setting:

$$
\begin{aligned}
& \Pi_{d} S_{d}=\bar{A}_{d} \Pi_{d}+B_{d} L_{d} \\
& \bar{C}_{d} \Pi_{d}+D_{d} L_{d}=Q_{d}
\end{aligned}
$$

with the following relation between $L$ and $L_{d}$ :

$$
L_{d}=\sqrt{2 \delta}\left(I-K(\delta I-A)^{-1} B\right)^{-1} L(\delta I-S)^{-1}
$$

Proof. The discrete control law that stabilizes the system and ensures the proper tracking of the discrete signal is given by: with the following relation between $L$ and $L_{d}$ :

$$
u_{k}=K_{d} x_{k-1}+L_{d} z_{k-1}
$$

The discrete error is defined as $e_{r, k}=x_{k}-\Pi_{d} z_{k}$, which leads to:

$$
e_{r, k}=x_{k}-\Pi_{d} z_{k}=\bar{A}_{d} x_{k-1}+B_{d} L_{d} z_{k-1}-\Pi_{d} z_{k}=\bar{A}_{d}\left[x_{k-1}-\Pi_{d} z_{k-1}\right]+\left[\bar{A}_{d} \Pi_{d}+B_{d} L_{d}-\Pi_{d} S_{d}\right] z_{k-1}
$$


and if the last term in brackets is equal zero, the error system becomes:

$$
e_{r, k}=\bar{A}_{d} e_{r, k-1}
$$

which is stable if $\bar{A}_{d}$ is stable. This yields the following Sylvester equation:

$$
\Pi_{d} S_{d}=\bar{A}_{d} \Pi_{d}+\bar{B}_{d} L_{d}
$$

And the tracking error will be:

$$
\begin{gathered}
e_{k}=y_{k}-y_{r, k}=\bar{C}_{d} x_{k-1}+D_{d} L_{d} z_{k-1}-Q_{d} z_{k-1}=\bar{C}_{d}\left[x_{k-1}-\Pi_{d} z_{k-1}\right]+\left[\bar{C}_{d} \Pi_{d} z_{k-1}+D_{d} L_{d} z_{k-1}-Q_{d} z_{k-1}\right]= \\
\bar{C}_{d} e_{k-1}+\left[\bar{C}_{d} \Pi_{d}+D_{d} L_{d}-Q_{d}\right] z_{k-1}
\end{gathered}
$$

which gives the condition:

$$
\bar{C}_{d} \Pi_{d}+\bar{D}_{d} L_{d}=Q_{d}
$$

Lastly, the proof that $\Pi_{d}(\zeta)=\Pi(\zeta)$ for the Cayley-Tustin time discretization is derived. First, the discrete Sylvester equation is considered and the definition of each operator is used:

$$
\begin{gathered}
\Pi_{d} S_{d}=\bar{A}_{d} \Pi_{d}+B_{d} L_{d} \rightarrow \\
\Pi_{d}(\delta I-S)^{-1}=(\delta I-A-B K)^{-1} \Pi_{d}+(\delta I-A)^{-1} B\left(I-K(\delta I-A)^{-1} B\right)^{-1} L(\delta I-S)^{-1} \rightarrow \\
\Pi_{d}(\delta I-S)^{-1}=(\delta I-A-B K)^{-1} \Pi_{d}+(\delta I-A-B K)^{-1} B L(\delta I-S)^{-1} \rightarrow \\
(\delta I-A-B K) \Pi_{d}=\Pi_{d}(\delta I-S)+B L \rightarrow \Pi_{d} S=(A+B K) \Pi_{d}+B L
\end{gathered}
$$

And for the algebraic condition:

$$
\begin{gathered}
\bar{C}_{d} \Pi_{d}+D_{d} L_{d}=Q_{d} \rightarrow \\
C(\delta I-A)^{-1}\left[I+B\left(I-K(\delta I-A)^{-1} B\right)^{-1} K(\delta I-A)^{-1}\right] \Pi_{d}+ \\
C\left(I-(\delta I-A)^{-1} B K\right)^{-1}(\delta I-A)^{-1} B L(\delta I-S)^{-1}=Q(\delta I-S)^{-1} \rightarrow \\
C(\delta I-A)^{-1}\left(I-B K(\delta I-A)^{-1}\right)^{-1} \Pi_{d}+C(\delta I-A-B K)^{-1} B L(\delta I-S)^{-1}=Q(\delta I-S)^{-1} \rightarrow \\
C\left[(\delta I-A-B K)^{-1} \Pi_{d}+(\delta I-A-B K)^{-1} B L(\delta I-S)^{-1}\right]=Q(\delta I-S)^{-1} \rightarrow \\
C \Pi_{d}(\delta I-S)^{-1}=Q(\delta I-S)^{-1} \rightarrow C \Pi_{d}=Q
\end{gathered}
$$

which is the same Sylvester equation and algebraic condition shown in the continuous-time setting (Equation (29)), consequently, $\Pi_{d}=\Pi$.

\subsection{Discrete System Observer Design}

The discrete observer design is considered in this section, as it was developed in Section 3.3 in the continuous time setting. In the continuous setting (Equation (43)), the observer does not have the same operator as the system $(\hat{A}$ is different from $A)$, thus, the discrete observer will also have different operators $A_{d}, B_{d}, C_{d}$ and $D_{d}$ when compared to the discrete system. Thus, the discrete system is observable as well and the discrete observer will take the following form:

$$
\begin{aligned}
& \hat{y}_{m, k}=\hat{C}_{m, d} \hat{x}_{k-1}+\hat{D}_{m, d} u_{k}+\left(M_{o d, 1}+M_{o d, 2}\right) y_{k} \\
& \hat{x}_{k}=\hat{A}_{d} \hat{x}_{k-1}+\hat{B}_{d} u_{k}+L_{o d, 1}\left[y_{m, k}-\hat{y}_{m, k}\right]+L_{o d, 2}\left[y_{m, k}\right]
\end{aligned}
$$


where $B_{d}$ and $y_{k}$ have been defined previously. The other discrete operators are given as:

$$
\begin{aligned}
& {\left[\begin{array}{cc}
\hat{A}_{d}(\cdot) & \hat{B}_{d} \\
\hat{C}_{m, d}(\cdot) & \hat{D}_{m, d}
\end{array}\right]=\left[\begin{array}{cc}
-I(\cdot)+2 \delta(\delta I-\hat{A})^{-1}(\cdot) & \sqrt{2 \delta}(\delta I-\hat{A})^{-1} B \\
\sqrt{2 \delta}\left[I+C_{m}(\delta I-\hat{A}) L_{o, 1}\right]^{-1} C_{m}(\delta I-\hat{A})^{-1} & {\left[I+C_{m}(\delta I-\hat{A}) L_{o, 1}\right]^{-1} C_{m}(\delta I-\hat{A})^{-1} B}
\end{array}\right]} \\
& M_{o d, 1}=\left[I+C_{m}(\delta I-\hat{A}) L_{o, 1}\right]^{-1} C_{m}(\delta I-\hat{A})^{-1} L_{o, 1} \quad L_{o d, 1}=\sqrt{2 \delta}(\delta I-\hat{A})^{-1} L_{o, 1} \\
& M_{o d, 2}=\left[I+C_{m}(\delta I-\hat{A}) L_{o, 1}\right]^{-1} C_{m}(\delta I-\hat{A})^{-1} L_{o, 2} \quad L_{o d, 2}=\sqrt{2 \delta}(\delta I-\hat{A})^{-1} L_{o, 2}
\end{aligned}
$$

Notice that $L_{o d, 1}$ and $L_{o d, 2}$ have similar structure to $B_{d}$, as the system measured output $y_{m, k}$ can be considered as an input to the observer as well.

Lemma 11. If the observer gains in the continuous time setting $\left(L_{0,1}\right.$ and $\left.L_{o, 2}\right)$ are chosen such that $\left(\hat{A}-L_{0,1} C_{m}\right)$ is stable, then, the discrete observer given by Equation (72) and the operators defined in Equation (73) will be able to reconstruct the states of the discrete system (the discrete observer error - $\hat{e}_{k}$-decreases with time and eventually reaches the origin).

Proof. To prove the observer states convergence to the system states, it is necessary to analyze the discrete observer error:

$$
\hat{e}_{k}=x_{k}-\hat{x}_{k}
$$

After some algebraic manipulation (shown in Appendix C), the discrete error can be written as:

$$
\hat{e}_{k}=\left(\hat{A}_{d}-L_{o d, 1} \hat{C}_{m, d}\right) \hat{e}_{k-1}=\left\{-I+2\left[\delta I-\hat{A}+L_{o, 1} C_{m}\right]^{-1}\right\} \hat{e}_{k-1}
$$

which is the discrete operator generated by $\left(\hat{A}-L_{o, 1} C_{m}\right)$. Thus, if $L_{o, 1}$ is chosen such that $\left(\hat{A}-L_{o, 1} C_{m}\right)$ is stable, the discrete observer will be stable as well, as the Cayley-Tustin time discretization cannot map a stable continuous system to a unstable discrete one.

\subsection{Discrete Exosystem Observer Design}

The discrete observer design for the exosystem is considered in this section using the discrete reference signal $y_{r, k}$. The following finite discrete observer is considered:

$$
\begin{gathered}
\hat{y}_{r, k}=\hat{Q}_{d} \hat{z}_{k-1}+M_{e d} y_{r, k} \\
\hat{z}_{k}=S_{d} \hat{z}_{k-1}+L_{e d}\left(y_{r, k}-\hat{y}_{r, k}\right)
\end{gathered}
$$

where $\hat{z}_{k}$ is the estimated exosystem state, $S_{d}$ and $Q_{d}$ have been defined in Section 4.3 (in Equation (60)). $L_{e d}$ and $M_{e d}$ are defined as:

$$
\begin{gathered}
L_{e d}=\sqrt{2 \delta}(\delta I-S)^{-1} L_{e} \\
\hat{Q}_{d}=\left[I+Q(\delta I-S)^{-1} L_{e}\right]^{-1} \sqrt{2 \delta} Q(\delta I-S)^{-1} \\
M_{e d}=\left[I+Q(\delta I-S)^{-1} L_{e}\right]^{-1} Q(\delta I-S)^{-1} L_{e}
\end{gathered}
$$

Lemma 12. If the exosystem observer gain in the continuous time setting $\left(L_{e}\right)$ is chosen such that $\left(S-L_{e} Q\right)$ is stable, then, the discrete observer given by Equation (A23) and the operators defined in Equation (A24) will be able to reconstruct the states of the discrete exosystem.

Proof. To prove the exosystem observer convergence to the system states, the discrete error of the exosystem observer is analyzed:

$$
\hat{e}_{r, k}=z_{k}-\hat{z}_{k}
$$


After some algebraic manipulation (shown in Appendix D), the discrete error can be written as:

$$
\hat{e}_{r, k}=\left(S_{d}-L_{e d} \hat{Q}_{d}\right) \hat{e}_{r, k-1}=\left\{-I+2\left[\delta I-S+L_{e} Q\right]^{-1}\right\} \hat{e}_{r, k-1}
$$

which is the operator generated by the discrete representation of $\left(S-L_{e} Q\right)$. Thus, if $L_{e}$ is chosen such that $\left(S-L_{e} Q\right)$ is stable, the discrete observer will be stable as well.

\subsection{Finite-Time Discrete Exosystem Observer Design}

The exosystem discrete dynamics is given by $z_{k}=S_{d} z_{k-1}$ and the reference signal is $y_{r, k}=Q_{d} z_{k-1}$. If the pair $\left(S_{d}, Q_{d}\right)$ is observable (which can be proved by the observability of $(S, Q)$ ), then the exosystem states can be estimated in the discrete setting using the observability matrix:

$$
\left[\begin{array}{c}
y_{r, k} \\
y_{r, k+1} \\
\vdots \\
y_{r, k+e-1}
\end{array}\right]=\left[\begin{array}{c}
Q_{d} \\
Q_{d} S_{d} \\
\vdots \\
Q_{d} S_{d}^{e-1}
\end{array}\right] \hat{z}_{k-1}=\operatorname{OBSV}_{E x o} \hat{z}_{k-1} \rightarrow \hat{z}_{k}=S_{d}^{k+1} \hat{z}_{k-1}
$$

where, $\mathrm{OBSV}_{E x o}$ is the exosystem observability matrix in the discrete-time setting. Therefore, after $e$ time steps (and $e$ samples of $y_{r, k}$ ) it is possible to properly estimate $z_{k}$. For any instance before that, the discrete observer from Section 4.5 can be used.

\section{Results}

In this section, the numerical simulations are shown to demonstrate the application of the regulator designed. The system parameters used in the following results are given in Table 1. For the given values the open-loop system is unstable, as shown in Section 2.1.1. The desired reference signal is given by the function:

$$
y(t)=1+0.1 t+\frac{\sin \left(\frac{\pi}{2} t\right)}{2}
$$

To generate this signal with the exosystem, the following conditions are chosen:

$$
Q=\left[\begin{array}{cccc}
1 & 0 & 1 & 0
\end{array}\right] ; f=\frac{1}{2} ; z(0)=\left[\begin{array}{cccc}
1 & 0.1 & 0 & 0.5
\end{array}\right]^{T}
$$

First, the simulations for the design of the regulator in the continuous-time setting are shown.

\subsection{Continuous Time Regulation}

Using the values given in Table 1, a numerical simulation was performed in the continuous time setting to show the system stabilization with the control law shown in Equation (22), in Section 3.1. The result is shown in Figure 5 and it is possible to see that the system is stabilized at a time close to $t=\phi_{1}+\phi_{2}=\frac{1}{\mu}+\max \left\{\frac{1}{\gamma_{1}}, \frac{1}{\gamma_{2}}\right\}=\frac{1}{2}+\max \left\{\frac{1}{0.5}, \frac{1}{1}\right\}=2.5$, as expected from the relation between the target and the closed-loop system.

Next, the results for the output tracking are presented. The result is shown in Figure 6 and the control law from Equation (10) is used. As expected from the finite time stabilization obtained due to the desired target system, the system output starts to perfectly track the reference signal for a time close to $t=2.5$. The results from Figures 5 and 6 consider that all the system states and exosystem states are available.

The measurement of all states is generally not feasible, specially when a DPS is considered. Thus, the control law shown in Equations (22) and (10) might not be used directly. Taking that into account, the observer design for the system states and the exosystem were considered in Sections 3.3 and 3.4, respectively. First, the results for the system observer are examined. Figure 7 shows the results for this simulation. As expected, the observer states are able to reconstruct the system states at a finite time close to $t=2.5$ (i.e., the observer error reaches the origin for a time close to $t=2.5$ ). 

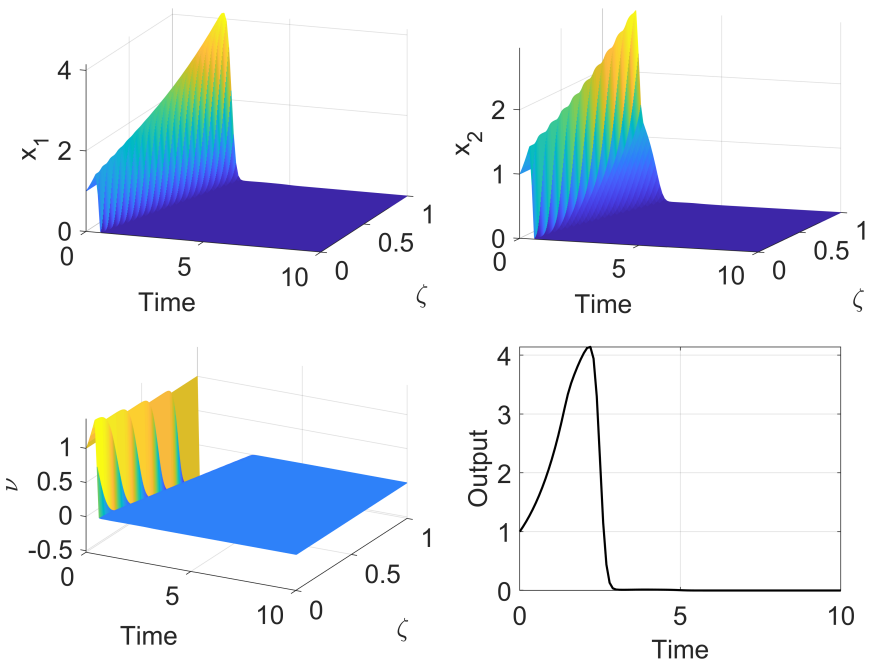

Figure 5. System stabilization with the control law given in Equation (22).
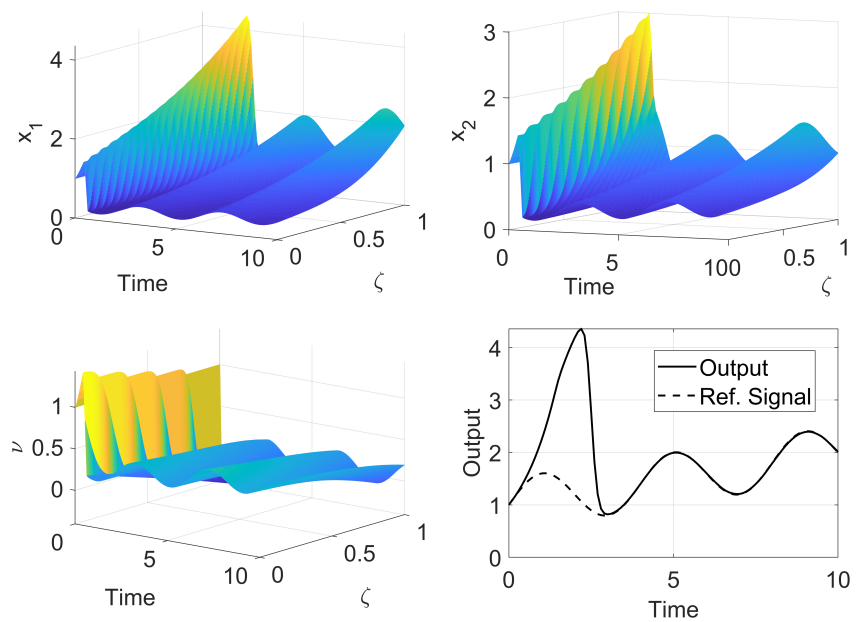

Figure 6. System stabilization and output regulation with the control law given in Equation (10).
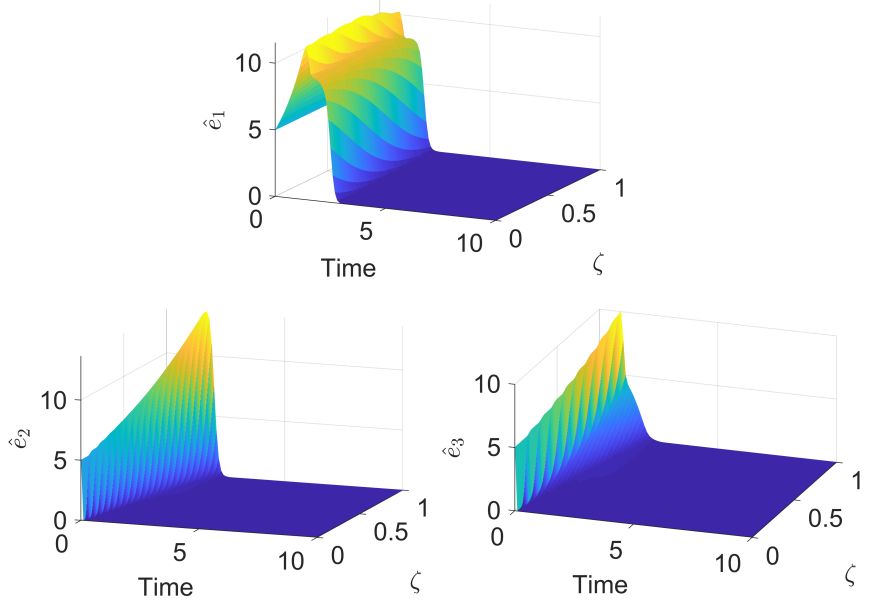

Figure 7. System observer error, using the observer developed in Section 3.3. 
Next, the performance of the system observer with the Equation (10) is examined. Instead of using the system states in the control law, the observer states are used and the result is shown in Figure 8. As the observer is able to properly reconstruct the system states for $t>t_{0}=2.5$, the controller can just properly stabilize and achieve proper tracking after that. Thus, the system is stabilized and the output properly tracks the reference signal at $t=t_{o}+t_{s}=5$.
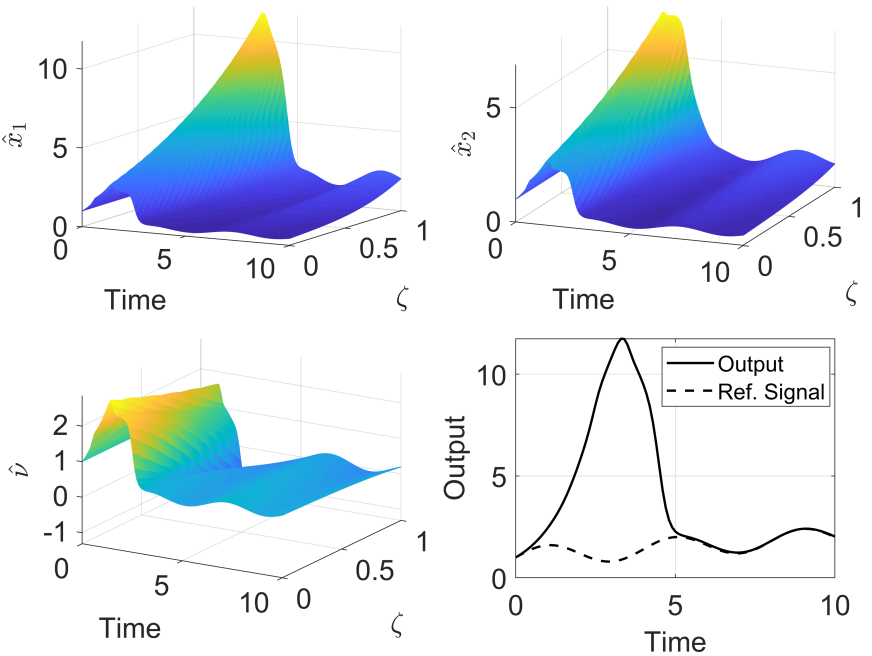

Figure 8. System closed-loop response, using the observer developed in Section 3.3 and the control law given in Equation (10).

The simulation results shown before still considered that the exosystem states were available. Next, the results with the exosystem observer, shown in Section 3.4, are explored. Figure 9 shows the closed-loop system response considering that the exosystem observer will have the poles placed at $\sigma\left(S-L_{e} Q\right)=\{-1,-2,-3,-4\}$. The finite stabilization and system observer convergence are still achieved, but, as the exosystem observer, in this case, does not have a finite time convergence, the proper output tracking takes longer to be obtained (it exponentially converges).
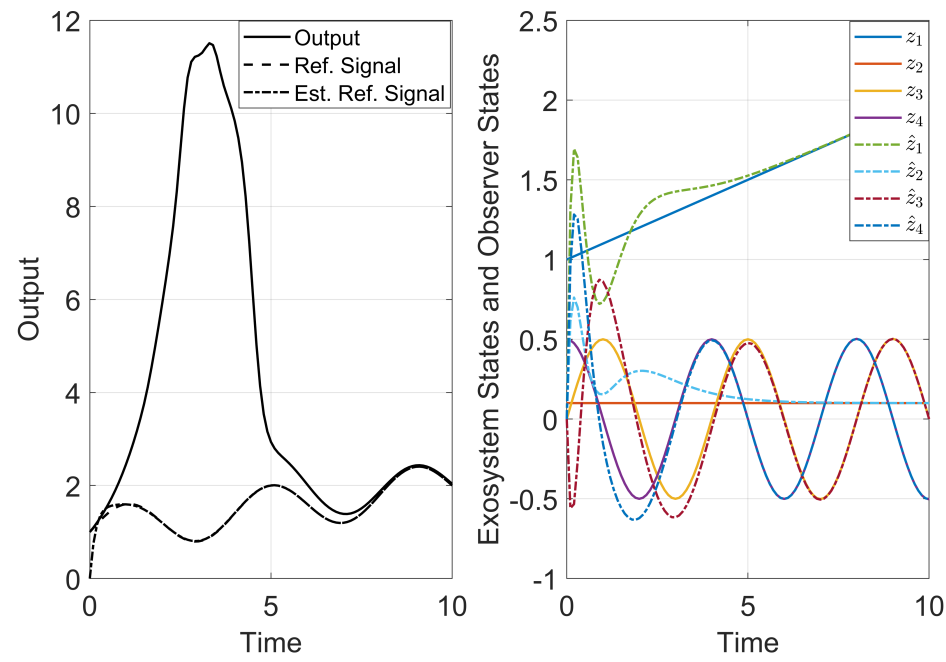

Figure 9. System closed-loop response (on the left), using the system and exosystem observers and the control law given in Equation (10). On the right, the exosystem states and its observer states.

The last case considered in the continuous-time setting is the use of the finite-time observer to reconstruct the exosystem states. The simulation results are presented in Figure 10. As two observer are 
necessary to obtain the finite-time convergence, one observer was designed such that $\sigma\left(S-L_{e, 1} Q\right)=$ $\{-1,-1.5,-2,-2.5\}$ and the other such that $\sigma\left(S-L_{e, 2} Q\right)=\{-3,-3.5,-4,-4.5\}$. The desired convergence time was set to $D_{F}=t_{o}+t_{s}=5$. Thus the system stability and proper tracking, plus the system and exosystem states reconstruction should be guaranteed for a time close to $t=5$. As results show, although this observer convergence is guaranteed for time close to $t=5$, the drawback is the higher error for the estimation in the beginning, which leads to excessive control actions and an increase in the output, which might not be desirable or physically possible.
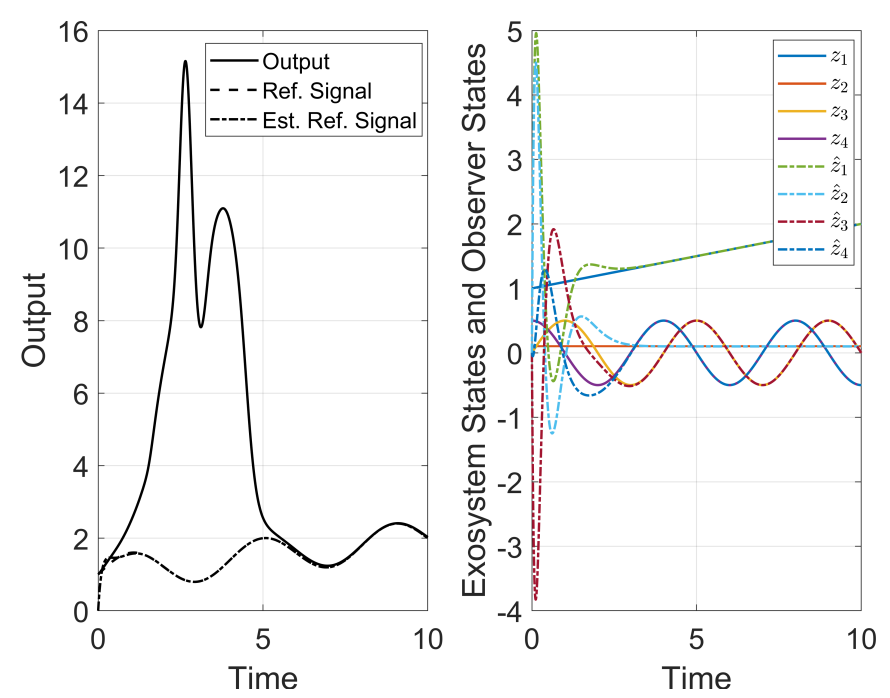

Figure 10. System closed-loop response (on the left), using the system observer, finite-time exosystem observer and the control law given in Equation (10). On the right, the exosystem states and its observer states.

\subsection{Discrete Time Regulation}

After the design of the regulator in the continuous-time setting has been achieved, the results for the discrete-time settings are considered, using the design developed in Section 4. A sampling time of $\Delta t=0.1$ is used. First, the system stabilization with the control law shown in Equation (53), developed in Section 4.2, is shown. The result is presented in Figure 11 and it is possible to see that the system is stabilized, as expected from the relation between the closed-loop of the discrete representation and the continuous closed-loop system.

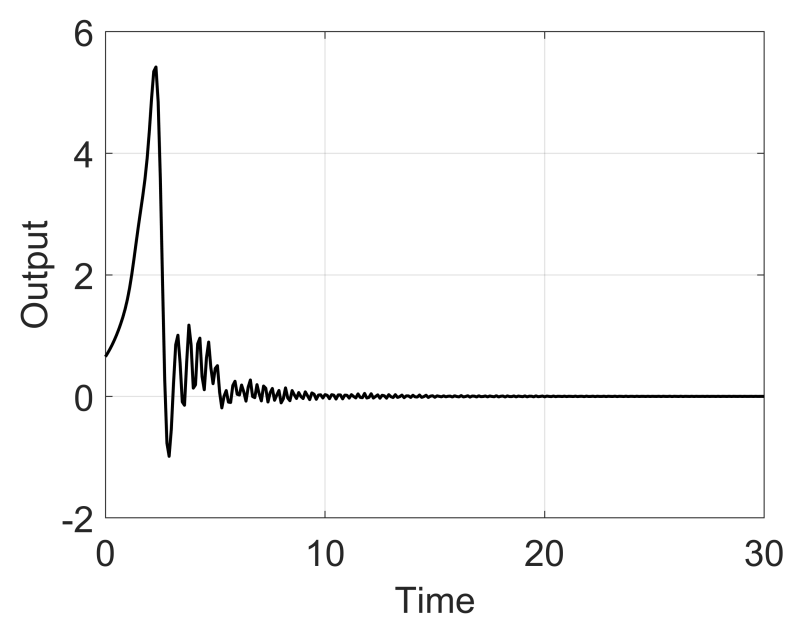

Figure 11. Discrete system stabilization with the control law given in Equation (53), considering $z_{k}=0$. 
Next, the results for the output tracking in the discrete time setting are considered. The result is shown in Figure 12 and the control law from Equation (53), developed in Section 4.3.1, is used. It is assumed that the system and exosystem states are known. As expected, the system stabilization is obtained and the system output is able to perfectly track the reference signal.

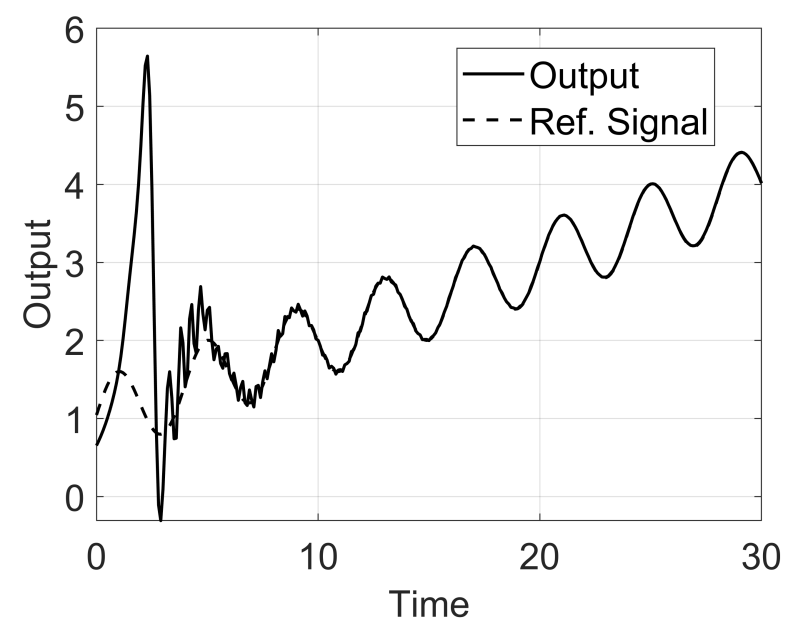

Figure 12. Discrete system stabilization and output regulation with the control law given in Equation (53), assuming that $z_{k}$ are known states.

As in the continuous-time setting, the system observer and exosystem observer designs in the discrete-time setting are considered next, using the developed discrete observers designs shown in Section 4.4 and Section 4.5, respectively. The results for the system observer are considered first in Figure 13. As expected, the observer is able to reconstruct the system states and using the observer states in the control law shown in Equation (53), the closed-loop of the discrete-time system is stable, achieving proper tracking of the reference signal.
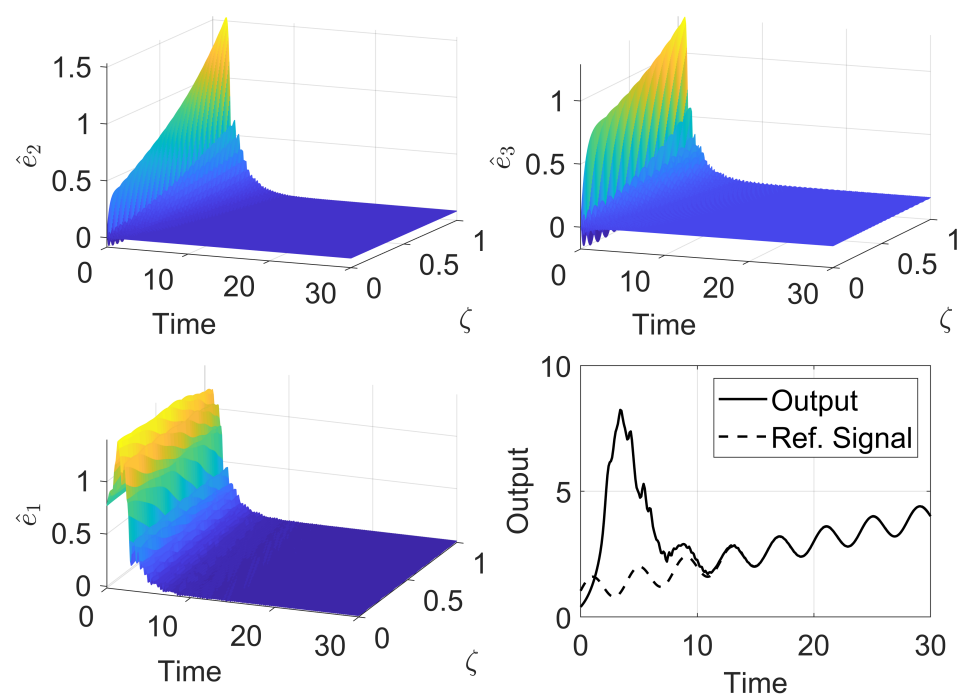

Figure 13. Discrete system closed-loop response, using the observer developed in Section 4.4 and the control law given in Equation (53).

Finally, the exosystem observer design in the discrete-time setting is considered, as shown in Section 4.5. Figure 14 shows the closed-loop response of the discrete system using the observer gain of the continuous-time setting to guarantee the stability of the observer in the discrete-time. As it is possible to notice, the system stabilization, the system and exosystem observer convergence are achieved, yielding to the proper tracking of the closed-loop system response. 

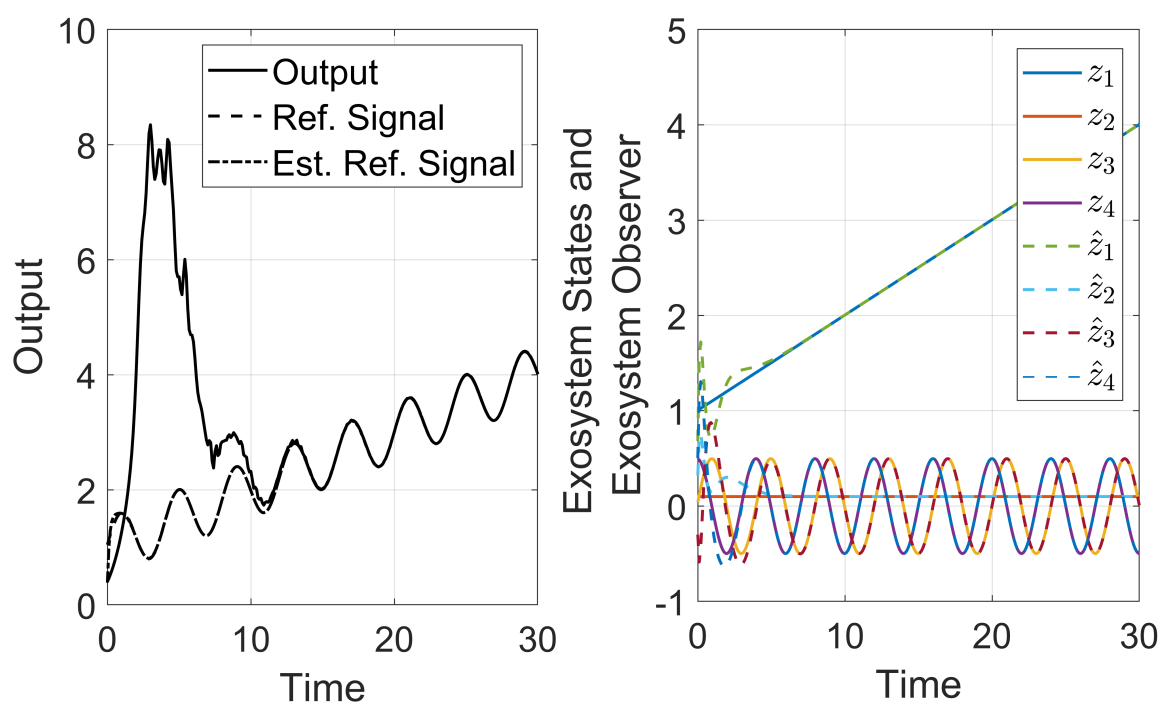

Figure 14. Discrete system closed-loop response (on the left), using the system and exosystem observers and the control law given in Equation (53). On the right, the discrete exosystem states and its observer states.

Lastly, the closed-loop response using a finite-time observer design in the discrete time setting based in the observability matrix is considered, as examined in Section 4.6. The result is shown in Figure 15 and, as expected, the exosystem states are properly reconstructed after four sampling times, as there are 4 states in the exosystem, which makes the closed-loop system response better.
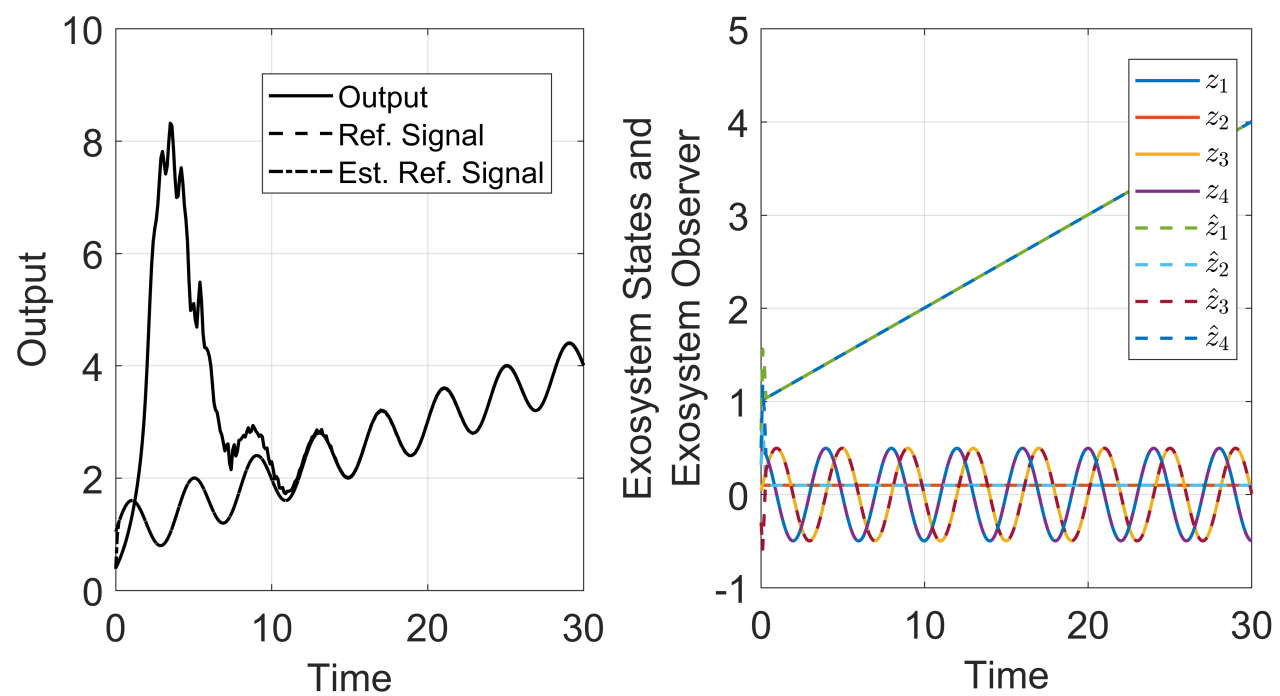

Figure 15. Discrete system closed-loop response (on the left), using the observability matrix to reconstruct the exosystem states. On the right, the discrete exosystem states and the observer states.

\section{Conclusions}

In this manuscript, the regulator design for the Saint-Venant-Exner model was developed so it would achieve proper closed-loop stability and output tracking of a reference signal in both continuous and discrete-time setting. The backstepping methodology was used in the continuous-time setting to map the closed-loop system to a stable target system, thus guaranteeing the system stability. Furthermore, the same method was used to design the observer, allowing for the reconstruction of the system states by using just the output. Considering a reference signal generated by an exosystem, the output tracking problem was solved. Next, with the stabilization and proper tracking achieved in 
the continuous-time setting, the discrete regulator was explored. The closed-loop stability, observer design and the regulator equations were shown to be directly related to their construction in the continuous-time, thus ensuring the proper performance of the regulator, as it was possible to observe in the simulations.

For future work, the constrained optimal stabilization and tracking problem could be considered, as the regulator designed here did not take into account any physical limitations, neither in the states nor the input.

Author Contributions: Conceptualization, G.O.C. and S.D.; Methodology, G.O.C. and S.D.; Supervision, S.D.; Writing, Original Draft, G.O.C.; Writing, Review and Editing, G.O.C. and S.D. All authors have read and agreed to the published version of the manuscript.

Funding: We acknowledge support provided by CAPES 88881.128514/2016-01 (Brazil) for Guilherme Ozorio Cassol.

Conflicts of Interest: The authors declare no conflict of interest.

\section{Appendix A. System Stabilization: Backstepping}

First, the state transformation $\omega(\zeta, t)=v(\zeta, t) \mathrm{e}^{\frac{\alpha_{1}}{\mu} \zeta}$ is used:

$$
\begin{aligned}
& \frac{\partial \omega}{\partial t}=\mu \frac{\partial \omega}{\partial \zeta} \quad+\theta_{1}(\zeta) x_{1}+\theta_{2}(\zeta) x_{2} \\
& \frac{\partial x_{1}}{\partial t}=-\gamma_{1} \frac{\partial x_{1}}{\partial \zeta}+\alpha(\zeta) \omega+\sigma_{11} x_{1} \quad+\sigma_{12} x_{2} \\
& \frac{\partial x_{2}}{\partial t}=-\gamma_{2} \frac{\partial x_{2}}{\partial \zeta}+\alpha(\zeta) \omega+\sigma_{21} x_{1} \quad+\sigma_{22} x_{2} \\
& \omega(1, t)=\rho_{1}^{\prime} x_{1}(1, t)+\rho_{2}^{\prime} x_{2}(1, t)+u^{\prime}(t) \\
& x_{1}(0, t)=q_{1} \omega(0, t) \\
& x_{2}(0, t)=q_{2} \omega(0, t) \\
& y_{m}(t)=v(0, t)=\omega(0, t) \\
& y(t)=x_{1}(1, t)
\end{aligned}
$$

where $\theta_{1}(\zeta)=\beta_{1} \mathrm{e}^{\frac{\alpha_{1}}{\mu} \zeta}, \theta_{2}(\zeta)=\beta_{2} \mathrm{e}^{\frac{\alpha_{1}}{\mu} \zeta}, \alpha(\zeta)=\alpha_{1} \mathrm{e}^{-\frac{\alpha_{1}}{\mu} \zeta}, \rho_{1}^{\prime}=\rho_{1} \mathrm{e}^{\frac{\alpha_{1}}{\mu} \zeta}, \rho_{2}^{\prime}=\rho_{2} \mathrm{e}^{\frac{\alpha_{1}}{\mu} \zeta}$ and $u^{\prime}(t)=u(t) \mathrm{e}^{\frac{\alpha_{1}}{\mu} \zeta}$.

The stabilization in the continuous-time setting was developed in [13] and assumes that the following Volterra transformation exists:

$$
\begin{aligned}
& W(\zeta, t)=\omega(\zeta, t)-\int_{0}^{\zeta}\left[K_{1}(\zeta, \eta) \omega(\eta, t)+K_{2}(\zeta, \eta) x_{1}(\eta, t)+K_{3}(\zeta, \eta) x_{2}(\eta, t)\right] d \eta \\
& X_{1}(\zeta, t)=x_{1}(\zeta, t) \\
& X_{2}(\zeta, t)=x_{2}(\zeta, t)
\end{aligned}
$$

$$
\left\{\begin{array}{l}
\mu \frac{\partial K_{1}}{\partial \zeta}+\mu \frac{\partial K_{1}}{\partial \eta}=\alpha(\eta) K_{2}+\alpha(\eta) K_{3} \\
\mu \frac{\partial K_{2}}{\partial \zeta}-\gamma_{1} \frac{\partial K_{2}}{\partial \eta}=\theta_{1}(\eta) K_{1}+\sigma_{11} K_{2}+\sigma_{21} K_{3} \\
\mu \frac{\partial K_{3}}{\partial \zeta}-\gamma_{2} \frac{\partial K_{3}}{\partial \eta}=\theta_{2}(\eta) K_{1}+\sigma_{12} K_{2}+\sigma_{22} K_{3} \\
\mu K_{1}(\zeta, 0)=q_{1} \gamma_{1} K_{2}(\zeta, 0)+q_{2} \gamma_{2} K_{3}(\zeta, 0) \\
K_{2}(\zeta, \zeta)=-\frac{\theta_{1}(\zeta)}{\gamma_{1}+\mu} \\
K_{3}(\zeta, \zeta)=-\frac{\theta_{2}(\zeta)}{\gamma_{2}+\mu}
\end{array}\right.
$$


The PDE system given in Equation (A3) can be solved numerically $[29,30]$. The control law and the Volterra transformation shown in (A2) map the system to the following target system:

$$
\left\{\begin{array}{l}
\frac{\partial W}{\partial t}=\mu \frac{\partial W}{\partial \zeta} \\
\frac{\partial X_{1}}{\partial t}=-\gamma_{1} \frac{\partial X_{1}}{\partial \zeta}+\int_{0}^{\zeta}\left[\kappa_{1}(\zeta, \eta) W(\eta, t)+c_{11}(\zeta, \eta) X_{1}(\eta, t)+c_{12}(\zeta, \eta) X_{2}(\eta, t)\right] d \eta \\
\frac{\partial X_{2}}{\partial t}=-\gamma_{2} \frac{\partial X_{2}}{\partial \zeta}-\int_{0}^{\zeta}\left[\kappa_{2}(\zeta, \eta) W(\eta, t)+c_{21}(\zeta, \eta) X_{1}(\eta, t)+c_{22}(\zeta, \eta) X_{2}(\eta, t)\right] d \eta \\
W(1, t)=r^{\prime}(t) \\
X_{1}(0, t)=q_{1} W(0, t) \\
X_{2}(0, t)=q_{2} W(0, t)
\end{array}\right.
$$

where $r^{\prime}(t)=r(t) \mathrm{e}^{\frac{\alpha_{1}}{\mu} \zeta}$ and the coefficients are defined as:

$$
\begin{aligned}
& c_{i j}(\zeta, \eta)=\alpha(\zeta) K_{j}(\zeta, \eta)+\int_{\eta}^{\zeta} \kappa_{i}(\zeta, \phi) K_{j}(\phi, \eta) d \phi \\
& \kappa_{i}(\zeta, \eta)=\alpha(\zeta) K_{3}(\zeta, \eta)+\int_{\eta}^{\zeta} \kappa_{i}(\zeta, \phi) K_{3}(\phi, \eta) d \phi
\end{aligned}
$$

\section{Appendix B. Observer Design: Backstepping}

The state transformation $\hat{\omega}(\zeta, t)=\hat{v}(\zeta, t) \mathrm{e}^{\frac{\alpha_{1}}{\mu} \zeta}$ is used:

$$
\begin{cases}\frac{\partial \hat{\omega}}{\partial t}= & \mu \frac{\partial \hat{\omega}}{\partial \zeta}+\theta_{1}(\zeta) \hat{x}_{1}+\theta_{2}(\zeta) \hat{x}_{2}-l_{1}(\zeta)\left[y_{m}(t)-\hat{\omega}(\zeta=0)\right] \\ \frac{\partial \hat{x}_{1}}{\partial t}= & -\gamma_{1} \frac{\partial \hat{x}_{1}}{\partial \zeta}+\alpha(\zeta) \omega+\sigma_{11} \hat{x}_{1}+\sigma_{12} \hat{x}_{2}-l_{2}(\zeta)\left[y_{m}(t)-\hat{\omega}(\zeta=0)\right] \\ \frac{\partial \hat{x}_{2}}{\partial t}= & -\gamma_{2} \frac{\partial \hat{x}_{2}}{\partial \zeta}+\alpha(\zeta) \omega+\sigma_{21} \hat{x}_{1}+\sigma_{22} \hat{x}_{2}-l_{3}(\zeta)\left[y_{m}(t)-\hat{\omega}(\zeta=0)\right] \\ \hat{\omega}(1, t)=\rho_{1}^{\prime} \hat{x}_{1}(1, t)+\rho_{2}^{\prime} \hat{x}_{2}(1, t)+u^{\prime}(t) \\ \hat{x}_{1}(0, t)=q_{1} y_{m}(t) \\ \hat{x}_{2}(0, t)=q_{2} y_{m}(t)\end{cases}
$$

where $\hat{x}(\zeta, t)=\left[\hat{\omega} \hat{x}_{1} \hat{x}_{2}\right]^{T}$ are the transformed observer states. The observer error $(\hat{e})$ is defined as $\hat{e}(\zeta, t)=\left[\hat{e}_{1} \hat{e}_{2} \hat{e}_{3}\right]=\left[\omega-\hat{\omega}, x_{1}-\hat{x}_{1}, x_{2}-\hat{x}_{2}\right]^{T} \in L^{2}(0,1)$, and the observer error dynamics is given as:

$$
\left\{\begin{array}{l}
\frac{\partial \hat{e}_{1}}{\partial t}=\mu \frac{\partial \hat{e}_{1}}{\partial \zeta}+\theta_{1}(\zeta) \hat{e}_{2}+\theta_{2}(\zeta) \hat{e}_{3}-l_{1}(\zeta) \hat{e}_{1}(\zeta=0) \\
\frac{\partial \hat{e}_{2}}{\partial t}=-\gamma_{1} \frac{\partial \hat{e}_{2}}{\partial \zeta}+\alpha(\zeta) \hat{e}_{1}+\sigma_{11} \hat{e}_{2}+\sigma_{12} \hat{e}_{3}-l_{2}(\zeta) \hat{e}_{1}(\zeta=0) \\
\frac{\partial \hat{e}_{3}}{\partial t}=-\gamma_{2} \frac{\partial \hat{e}_{3}}{\partial \zeta}+\alpha(\zeta) \hat{e}_{1}+\sigma_{21} \hat{e}_{2}+\sigma_{22} \hat{e}_{3}-l_{3}(\zeta) \hat{e}_{1}(\zeta=0) \\
\hat{e}_{1}(1, t)=\rho_{1}^{\prime} \hat{e}_{2}(1, t)+\rho_{2}^{\prime} \hat{e}_{3}(1, t) \\
\hat{e}_{2}(0, t)=0 \\
\hat{e}_{3}(0, t)=0
\end{array}\right.
$$

which can be represented in a general form as:

$$
\dot{\hat{e}}(t)=\left(\hat{A}-L_{o, 1} C_{m}\right) \hat{e}(t)
$$

Therefore, to design the observer it is necessary to find an appropriate set of gains $L_{1}$ that makes $\hat{A}-L_{o, 1} C_{m}$ stable. Considering the error system shown in Equation (A7), the following stable target system is desired: 


$$
\left\{\begin{array}{l}
\frac{\partial \tilde{e}_{1}}{\partial t}=\mu \frac{\partial \tilde{e}_{1}}{\partial \zeta}+\theta_{1}(\zeta) \tilde{e}_{2}+\theta_{2}(\zeta) \tilde{e}_{3}+\int_{0}^{\zeta} \hat{g}_{12}(\zeta, \eta) \tilde{e}_{2}(\eta) d \eta+\int_{0}^{\zeta} \hat{g}_{13}(\zeta, \eta) \tilde{e}_{3}(\eta) d \eta \\
\frac{\partial \tilde{e}_{2}}{\partial t}=-\gamma_{1} \frac{\partial \tilde{e}_{2}}{\partial \zeta}+\sigma_{11} \tilde{e}_{2}+\sigma_{12} \tilde{e}_{3}+\int_{0}^{\zeta} \hat{g}_{22}(\zeta, \eta) \tilde{e}_{2}(\eta) d \eta+\int_{0}^{\zeta} \hat{g}_{23}(\zeta, \eta) \tilde{e}_{3}(\eta) d \eta \\
\frac{\partial \tilde{e}_{3}}{\partial t}=-\gamma_{2} \frac{\partial \tilde{e}_{3}}{\partial \zeta}+\sigma_{21} \tilde{e}_{2}+\sigma_{22} \tilde{e}_{3}+\int_{0}^{\zeta} \hat{g}_{32}(\zeta, \eta) \tilde{e}_{2}(\eta) d \eta+\int_{0}^{\zeta} \hat{g}_{33}(\zeta, \eta) \tilde{e}_{3}(\eta) d \eta \\
\tilde{e}_{1}(1, t)=\rho_{1}^{\prime} \tilde{e}_{e}(1, t)+\rho_{2}^{\prime} \tilde{e}_{3}(1, t) \\
\tilde{e}_{2}(0, t)=0 \\
\tilde{e}_{3}(0, t)=0
\end{array}\right.
$$

To achieve the target system, the following backstepping transformation is used:

$$
\tilde{e}_{i}(\zeta, t)=\hat{e}_{i}(\zeta, t)+\int_{0}^{\zeta} m_{i}(\zeta, \eta) \hat{e}_{1}(\zeta, t) d \eta
$$

For $i=1,2,3$, where $m_{i}(\zeta, \eta)$ are the transformation kernels, which can be found by solving the following set of equations:

$$
\left\{\begin{array}{l}
\mu \frac{\partial m_{1}}{\partial \zeta}+\mu \frac{\partial m_{1}}{\partial \eta}=-\theta_{1}(\zeta) K_{2}-\theta_{2}(\zeta) K_{3} \\
\gamma_{1} \frac{\partial m_{2}}{\partial \zeta}-\mu \frac{\partial m_{2}}{\partial \eta}=\alpha(\zeta) m_{1}+\sigma_{11} m_{2}+\sigma_{21} m_{3} \\
\gamma_{2} \frac{\partial m_{3}}{\partial \zeta}-\mu \frac{\partial m_{3}}{\partial \eta}=\alpha(\zeta) m_{1}+\sigma_{12} m_{2}+\sigma_{22} m_{3} \\
m_{1}(1, \eta)=\rho_{1} m_{2}(1, \eta)+\rho_{2} m_{3}(1, \eta) \\
m_{2}(\zeta, \zeta)=\frac{\alpha(\zeta)}{\gamma_{1}+\mu} \\
m_{3}(\zeta, \zeta)=\frac{\alpha(\zeta)}{\gamma_{2}+\mu}
\end{array}\right.
$$

This system can be solved numerically in the domain $0 \leq \eta \leq \zeta \leq 1$, and result in the following equations for the functions $\hat{g}_{i, j}$, for $i=1,2,3$ and $j=2,3$ in Equation (A9):

$$
\hat{g}_{i, j}=-\theta_{j}(\zeta) m_{i}(\zeta, \eta)-\int_{\eta}^{\zeta} m_{i}(\zeta, \phi) \hat{g}_{1, j}(\phi, \eta) d \phi
$$

And the observer gains $l_{i}(\zeta)$ will be given as:

$$
l_{i}(\zeta)=-\mu m_{i}(\zeta, \eta=0)
$$

\section{Appendix C. Discrete System Observer Stability}

It is necessary to show that the observer error can be written as:

$$
\hat{e}_{k}=x_{k}-\hat{x}_{k}=\left(\hat{A}_{d}-L_{o d, 1} \hat{C}_{m, d}\right) \hat{e}_{k-1}
$$

First, the relation between the system discrete operators and the observer operators is defined. As $A=\hat{A}+L_{o, 2} C_{m}$ the following relation between $(\delta I-A)^{-1}$ and $(\delta I-\hat{A})^{-1}$ holds:

$$
(\delta I-A)^{-1}=\left(\delta I-\hat{A}-L_{0,2} C_{m}\right)^{-1}=(\delta I-\hat{A})^{-1}+(\delta I-\hat{A})^{-1} L_{o, 2}\left[I-C_{m}(\delta I-\hat{A})^{-1} L_{0,2}\right]^{-1} C_{m}(\delta I-\hat{A})^{-1}
$$

Thus, the following relations between the system discrete operators and the observer operators will hold as well: 


$$
\begin{gathered}
A_{d}=\hat{A}_{d}+L_{o d, 2}\left[I-C_{m}(\delta I-\hat{A})^{-1} L_{o, 2}\right]^{-1} \sqrt{2 \delta} C_{m}(\delta I-\hat{A})^{-1} \\
B_{d}=\hat{B}_{d}+L_{o d, 2}\left[I-C_{m}(\delta I-\hat{A})^{-1} L_{o, 2}\right]^{-1} C_{m}(\delta I-\hat{A})^{-1} B \\
C_{m, d}=\left[I-C_{m}(\delta I-\hat{A})^{-1} L_{o, 2}\right]^{-1} \sqrt{2 \delta} C_{m}(\delta I-\hat{A})^{-1}=\left[I-C_{m}(\delta I-\hat{A})^{-1} L_{o, 2}\right]^{-1}\left[I+C_{m}(\delta I-\hat{A})^{-1} L_{o, 1}\right] \hat{C}_{m, d} \\
D_{m, d}=\left[I-C_{m}(\delta I-\hat{A})^{-1} L_{o, 2}\right]^{-1} C_{m}(\delta I-\hat{A})^{-1} B=\left[I-C_{m}(\delta I-\hat{A})^{-1} L_{o, 2}\right]^{-1}\left[I+C_{m}(\delta I-\hat{A})^{-1} L_{o, 1}\right] \hat{D}_{m, d}
\end{gathered}
$$

And the discrete observer error can be written as:

$$
\begin{gathered}
\hat{e}_{k}=x_{k}-\hat{x}_{k}=A_{d} x_{k-1}+B_{d} u_{k}-\left(\hat{A}_{d} \hat{x}_{k-1}+\hat{B}_{d} u_{k}+L_{o d, 1}\left[y_{m, k}-\hat{y}_{m, k}\right]+L_{o d, 2}\left[y_{m, k}\right]\right)= \\
\hat{A}_{d}\left(x_{k-1}-\hat{x}_{k-1}\right)+L_{o d, 2}\left[I-C_{m}(\delta I-\hat{A})^{-1} L_{o, 2}\right]^{-1} \sqrt{2 \delta} C_{m}(\delta I-\hat{A})^{-1} x_{k-1} \\
+L_{o d, 2}\left[I-C_{m}(\delta I-\hat{A})^{-1} L_{o, 2}\right]^{-1} C_{m}(\delta I-\hat{A})^{-1} B u_{k}-\left(L_{o d, 1}\left[y_{m, k}-\hat{y}_{m, k}\right]+L_{o d, 2}\left[y_{m, k}\right]\right)
\end{gathered}
$$

By the definition of the operators, it is possible to write:

$$
y_{m, k}=C_{m, d} x_{k-1}-D_{m, d} u_{k}=\left[I-C_{m}(\delta I-\hat{A})^{-1} L_{o, 2}\right]^{-1}\left[\sqrt{2 \delta} C_{m}(\delta I-\hat{A})^{-1} x_{k-1}+C_{m}(\delta I-\hat{A})^{-1} B u_{k}\right]
$$

and:

$$
\begin{gathered}
{\left[y_{m, k}-\hat{y}_{m, k}\right]=-\hat{C}_{m, d} \hat{x}_{k-1}-\hat{D}_{m, d} u_{k}+\left(I-M_{o d, 1}-M_{o d, 2}\right) y_{k}=} \\
-\hat{C}_{m, d} \hat{x}_{k-1}-\hat{D}_{m, d} u_{k}+\left(I-M_{o d, 1}-M_{o d, 2}\right)\left[I-C_{m}(\delta I-\hat{A})^{-1} L_{o, 2}\right]^{-1}\left[I+C_{m}(\delta I-\hat{A})^{-1} L_{o, 1}\right]\left(\hat{C}_{m, d} x_{k-1}+\hat{D}_{m, d} u_{k}\right) \rightarrow \\
\left(I-M_{o d, 1}-M_{o d, 2}\right)=\left[I+C_{m}(\delta I-\hat{A}) L_{o, 1}\right]^{-1}\left[I+C_{m}(\delta I-\hat{A}) L_{o, 1}-C_{m}(\delta I-\hat{A})^{-1} L_{o, 1}-C_{m}(\delta I-\hat{A})^{-1} L_{o, 2}\right] \\
=\left[I+C_{m}(\delta I-\hat{A}) L_{o, 1}\right]^{-1}\left[I-C_{m}(\delta I-\hat{A})^{-1} L_{o, 2}\right] \rightarrow \\
\left(I-M_{o d, 1}-M_{o d, 2}\right)\left[I-C_{m}(\delta I-\hat{A})^{-1} L_{o, 2}\right]^{-1}=\left[I+C_{m}(\delta I-\hat{A}) L_{o, 1}\right]^{-1} \rightarrow \\
{\left[y_{m, k}-\hat{y}_{m, k}\right]=-\hat{C}_{m, d} \hat{x}_{k-1}-\hat{D}_{m, d} u_{k}+\left[I+C_{m}(\delta I-\hat{A}) L_{o, 1}\right]^{-1}\left[I+C_{m}(\delta I-\hat{A})^{-1} L_{o, 1}\right]\left(\hat{C}_{m, d} x_{k-1}+\hat{D}_{m, d} u_{k}\right)=} \\
\hat{C}_{m, d}\left(x_{k-1}-\hat{x}_{k-1}\right)
\end{gathered}
$$

Finally, the discrete observer error can be written as:

$$
\hat{e}_{k}=\hat{A}_{d}\left(x_{k-1}-\hat{x}_{k-1}\right)-L_{o d, 1} \hat{C}_{m, d}\left(x_{k-1}-\hat{x}_{k-1}\right)=\left(\hat{A}_{d}-L_{o d, 1} \hat{C}_{m, d}\right) \hat{e}_{k-1}
$$

And this can be linked to the operators in the continuous time setting by:

$$
\begin{gathered}
\hat{A}_{d}-L_{o d, 1} \hat{C}_{m, d}=-I+2 \delta(\delta I-\hat{A})^{-1}-2 \delta\left\{I-\left[I+(\delta I+\hat{A})^{-1} L_{o, 1} C_{m}\right]^{-1}\right\}(\delta I-\hat{A})^{-1}= \\
-I+2 \delta\left[I+(\delta I-\hat{A})^{-1} L_{o, 1} C_{m}\right]^{-1}(\delta I-\hat{A})^{-1}=-I+2\left[\delta I-\hat{A}+L_{o, 1} C_{m}\right]^{-1}
\end{gathered}
$$

which would be the discrete operator generated by $\left(\hat{A}-L_{0,1} C_{m}\right)$. Thus, if $L_{o, 1}$ is chosen such that $(\hat{A}-$ $\left.L_{o, 1} C_{m}\right)$ is stable, the discrete observer will be stable as well, as the Cayley-Tustin time discretization cannot map a stable continuous system to a unstable discrete one.

\section{Appendix D. Discrete Exosystem Observer Stability}

Similarly to the system observer, it is necessary to show that the exosystem observer error can be written as:

$$
\begin{gathered}
\hat{e}_{r, k}=z_{k}-\hat{z}_{k}=\left(S_{d}-L_{e d} Q_{d}\right) \hat{e}_{r, k-1} \\
\hat{y}_{r, k}=\hat{Q}_{d} \hat{z}_{k-1}+M_{e d} y_{r, k} \\
\hat{z}_{k}=S_{d} \hat{z}_{k-1}-L_{e d}\left(y_{r, k}-\hat{y}_{r, k}\right)
\end{gathered}
$$


where $\hat{z}_{k}$ is the estimated exosystem state, $S_{d}$ and $Q_{d}$ have been defined in Section 4.3 (in Equation (60)). $L_{e d}$ and $M_{e d}$ are defined as:

$$
\begin{gathered}
L_{e d}=\sqrt{2 \delta}(\delta I-S)^{-1} L_{e} \\
M_{e d}=\left[I+Q(\delta I-S)^{-1} L_{e}\right]^{-1} Q(\delta I-S)^{-1} L_{e}
\end{gathered}
$$

Lemma A1. If the exosystem observer gain in the continuous time setting $\left(L_{e}\right)$ is chosen such that $\left(S-L_{e} Q\right)$ is stable, then, the discrete observer given by Equation (A23) and the operators defined in Equation (A24) will be able to reconstruct the states of the discrete exosystem.

Proof. To prove the exosystem observer convergence to the system states, the discrete error of the exosystem observer is analyzed:

$$
\hat{e}_{r, k}=z_{k}-\hat{z}_{k}
$$

After some algebraic manipulation (shown in Appendix D), the discrete error can be written as:

$$
\hat{e}_{r, k}=\left(S_{d}-L_{e d} \hat{Q}_{d}\right) \hat{e}_{r, k-1}=\left\{-I+2\left[\delta I-S+L_{e} Q\right]^{-1}\right\} \hat{e}_{r, k-1}
$$

which is the discrete operator generated by $\left(S-L_{e} Q\right)$. Thus, if $L_{e}$ is chosen such that $\left(S-L_{e} Q\right)$ is stable, the discrete observer will be stable as well.

First, the discrete observer error can be written as:

$$
\begin{gathered}
\hat{e}_{r, k}=z_{k}-\hat{z}_{k}=S_{d} z_{k-1}-S_{d} \hat{z}_{k-1}-L_{e d}\left(y_{r, k}-\hat{y}_{r, k}\right)=S_{d}\left(z_{k-1}-\hat{z}_{k-1}\right)-L_{e d}\left(\left(I-M_{e d}\right) Q_{d} z_{k-1}-\hat{Q}_{d} \hat{z}_{k-1}\right) \rightarrow \\
\left(I-M_{e d}\right) Q_{d}=\left[I+Q(\delta I-S)^{-1} L_{e}\right]^{-1}\left[I+Q(\delta I-S)^{-1} L_{e}-Q(\delta I-S)^{-1} L_{e}\right] Q_{d} \\
=\left[I+Q(\delta I-S)^{-1} L_{e}\right]^{-1} Q_{d}=\hat{Q}_{d} \rightarrow=S_{d}\left(z_{k-1}-\hat{z}_{k-1}\right)-L_{e d}\left(\hat{Q}_{d} z_{k-1}-\hat{Q}_{d} \hat{z}_{k-1}\right)=\left(S_{d}-L_{e d} \hat{Q}_{d}\right) \hat{e}_{r, k-1}
\end{gathered}
$$

And this can be linked to the operators in the continuous time setting by:

$$
\begin{gathered}
S_{d}-L_{e d} \hat{Q}_{d}=-I+2 \delta(\delta I-S)^{-1}-2 \delta\left\{I-\left[I+(\delta I+S)^{-1} L_{e} Q\right]^{-1}\right\}(\delta I-S)^{-1}= \\
-I+2 \delta\left[I+(\delta I-S)^{-1} L_{e} Q\right]^{-1}(\delta I-S)^{-1}=-I+2\left[\delta I-S+L_{e} Q\right]^{-1}
\end{gathered}
$$

which would be the discrete operator generated by $\left(S-L_{e} Q\right)$. Choosing $L_{e}$ such that $\left(S-L_{e} Q\right)$ is stable will make the discrete exosystem observer stable as well if the exosystem observer shown in Section 3.4 is used.

\section{References}

1. Curtain, R.F.; Zwart, H. An Introduction to Infinite-Dimensional Linear Systems Theory; Springer: New York, NY, USA, 1995.

2. Bensoussan, A.; Prato, G.; Delfour, M.; Mitter, S. Representation and Control of Infinite Dimensional Systems; Birkhauser: Boston, MA, USA, 2007.

3. Alizadeh Moghadam, A.; Aksikas, I.; Dubljevic, S.; Forbes, J.F. Boundary optimal (LQ) control of coupled hyperbolic PDEs and ODEs. Automatica 2013, 49, 526-533. [CrossRef]

4. Byrnes, C.I.; Lauko, I.G.; Gilliam, D.S.; Shubov, V.I. Output regulation for linear distributed parameter systems. IEEE Trans. Autom. Control 2000, 2, 170-194.

5. Natarajan, V.; Gilliam, D.S.; Weiss, G. The State Feedback Regulator Problem for Regular Linear Systems. IEEE Trans. Autom. Control 2014, 59, 2708-2723. [CrossRef]

6. $\mathrm{Xu}, \mathrm{X}$.; Dubljevic, S. Finite-dimensional output feedback regulator for a mono-tubular heat exchanger process. IFAC-PapersOnLine 2016, 49, 54-59. [CrossRef] 
7. Hämäläinen, T.; Pohjolainen, S. Robust Regulation of Distributed Parameter Systems with Infinite-Dimensional Exosystems. SIAM J. Control Optim. 2010, 48, 4846-4873. [CrossRef]

8. Paunonen, L.; Pohjolainen, S. Internal Model Theory for Distributed Parameter Systems. SIAM J. Control Optim. 2010, 48, 4753-4775. [CrossRef]

9. Paunonen, L.; Pohjolainen, S. Robust controller design for infinite-dimensional exosystems. Int. J. Robust Nonlinear Control 2014, 24, 825-858. [CrossRef]

10. Xu, C.Z.; Sallet, G. Proportional and Integral Regulation of Irrigation canal Systems governed by the St Venant Equation. IFAC Proc. Vol. 1999, 32, 2274-2279. [CrossRef]

11. Litrico, X.; Fromion, V. H/sub /spl infin// control of an irrigation canal pool with a mixed control politics. IEEE Trans. Control Syst. Technol. 2006, 14, 99-111. [CrossRef]

12. Prieur, C.; de Halleux, J. Stabilization of a 1-D tank containing a fluid modeled by the shallow water equations. Syst. Control Lett. 2004, 52, 167-178. [CrossRef]

13. Diagne, A.; Diagne, M.; Tang, S.; Krstic, M. Backstepping stabilization of the linearized Saint-Venant-Exner model. Automatica 2017, 76, 345-354. [CrossRef]

14. Aamo, O. Disturbance Rejection in 2x2 Linear Hyperbolic Systems. IEEE Trans. Autom. Control 2013, 58, 1095-1106. [CrossRef]

15. Deutscher, J. Output regulation for linear distributed-parameter systems using finite-dimensional dual observers. Automatica 2011, 47, 2468-2473. [CrossRef]

16. Mlayeh, R.; Toumi, S.; Beji, L. Backstepping boundary observer based-control for hyperbolic PDE in rotary drilling system. Appl. Math. Comput. 2018, 322, 66-78. [CrossRef]

17. Deutschmann, A.; Jadachowski, L.; Kugi, A. Backstepping-based boundary observer for a class of time-varying linear hyperbolic PIDEs. Automatica 2016, 68, 369-377. [CrossRef]

18. Deutscher, J.; Gehring, N.; Kern, R. Output feedback control of general linear heterodirectional hyperbolic ODE-PDE-ODE systems. Automatica 2018, 95, 472-480. [CrossRef]

19. Karafyllis, I.; Krstic, M. Sampled-data boundary feedback control of 1-D linear transport PDEs with non-local terms. Syst. Control Lett. 2017, 107, 68-75. [CrossRef]

20. Karafyllis, I.; Krstic, M. Sampled-data boundary feedback control of 1-D parabolic PDEs. Automatica 2018, 87, 226-237. [CrossRef]

21. Kazantzis, N.; Kravaris, C. Energy-predictive control: A new synthesis approach for nonlinear process control. Chem. Engn. Sci. 1999, 54, 1697-1709. [CrossRef]

22. Hairer, E.; Lubich, C.; Wanner, G. Geometric Numerical Integration: Structure-Preserving Algorithms for Ordinary Differential Equations; Springer Series in Computational Mathematics; Springer: Berlin, Germany, 2002; Volume 31.

23. Havu, V.; Malinen, J. The Cayley transform as a time discretization scheme. Numer. Funct. Anal. Optim. 2007, 28, 825-851. [CrossRef]

24. Engel, R.; Kreisselmeier, G. A continuous-time observer which converges in finite time. IEEE Trans. Autom. Control 2002, 47, 1202-1204. [CrossRef]

25. Deutscher, J. Finite-time output regulation for linear $2 \times 2$ hyperbolic systems using backstepping. Automatica 2017, 75, 54-62. [CrossRef]

26. Dubljevic, S.; Humaloja, J.P. Model Predictive Control for Regular Linear Systems. arXiv 2018, arXiv:0706.1234.

27. Besseling, N. Stability analysis in continuous and discrete time. Ph.D. Thesis, University of Twente, Enschede, The Netherlands, 2012.

28. Xu, Q.; Dubljevic, S. Linear Model Predictive Control for Transport-Reaction Processes. AIChE J. 2017, 63, 2644-2659. [CrossRef]

29. Izadi, M.; Abdollahi, J.; Dubljevic, S.S. PDE backstepping control of one-dimensional heat equation with time-varying domain. Automatica 2015, 54, 41-48. [CrossRef]

30. Backstepping output-feedback control of moving boundary parabolic PDEs. Eur. J. Control 2015, 21, 27-35. [CrossRef]

(C) 2020 by the authors. Licensee MDPI, Basel, Switzerland. This article is an open access article distributed under the terms and conditions of the Creative Commons Attribution (CC BY) license (http:/ / creativecommons.org/licenses/by/4.0/). 